\title{
A nonsmooth frictional contact formulation for multibody system dynamics
}

\author{
Javier Galvez ${ }^{1}$, Federico J. Cavalieri ${ }^{2}$, Alejandro Cosimo ${ }^{1,2}$, Olivier \\ Brüls $^{1}$ and Alberto Cardona ${ }^{2}$ \\ ${ }^{1}$ University of Liège, Department of Aerospace and Mechanical \\ Engineering, Allée de la Découverte 9 (B52), 4000 Liège, Belgium \\ ${ }^{2}$ Universidad Nacional del Litoral - CONICET, Centro de \\ Investigación de Métodos Computacionales (CIMEC), Col. Ruta Nac \\ 168 s/n, predio CONICET, 3000 Santa Fe, Argentina
}

\begin{abstract}
We present a new node-to-face frictional contact element for the simulation of the nonsmooth dynamics of systems composed of rigid and flexible bodies connected by kinematic joints. The equations of motion are integrated using a nonsmooth generalized- $\alpha$ time integration scheme and the frictional contact problem is formulated using a mixed approach, based on an augmented Lagrangian technique and a Coulomb friction law. The numerical results are independent of any user-defined penalty parameter for the normal or tangential component of the forces and, the bilateral and the unilateral constraints are exactly fulfilled both at position and velocity levels. Finally, the robustness and the performance of the proposed algorithm are demonstrated by solving several numerical examples of nonsmooth mechanical systems involving frictional contact.
\end{abstract}

Keywords: Solids; Impact; Nonlinear dynamics; Contact; Multibody dynamics; Time integration, Implicit

\section{Introduction}

Many mechanical engineering applications are subjected to high frequency vibrations produced by impacts between components that can reduce the service life of the entire system, for example: robotic manipulators [1], gearboxes [2] and electrical circuit breakers [3]. Thus, an important challenge to design this kind of mechanisms, is the accurate prediction of velocities, accelerations and forces that occur in a short period of time. Over the past few decades, several authors have proposed numerical techniques to 
deal with the highly nonlinear system of equations resulting from the Signorini condition and the Coulomb friction law, which are usually used to model problems involving impacts and friction. However, there is not yet a completely robust algorithm suitable for a large range of applications dealing with impacts and friction, so that contact mechanics is still an active research area even nowadays.

The first mathematical formulation for frictional contact problems was proposed more than 200 years ago by Coulomb and then followed by Hertz [4]. In this law, only one macroscopic parameter is considered in the formulation: the friction coefficient. Although this law is very simple, it allows one to represent with an acceptable accuracy a wide range of real world applications. Since this seminal work, great efforts were made to better represent the friction process by including tribological properties, such as the lubrication conditions, the plastic deformations and the geometric changes of the contact surfaces, at the cost of an increased difficulty of the analysis. Hence, the Coulomb friction law is still commonly used for system-level simulation, because of its simplicity and its ability to capture essential aspects of the friction process. In numerical formulations, when the Coulomb friction law is used, the normal force is independent of the friction force, and, consequently, a non-symmetric system of equations is obtained. This results in an increase in the complexity of the numerical solver and in a degradation of the convergence rate of the algorithms $[5,6]$. A compilation of standard frictional contact formulations can be found in [7-10].

In computational contact mechanics, the enforcement of the unilateral constraint of the frictional contact/impact problem can be addressed by three main strategies: penalty, Lagrange multipliers, and augmented Lagrangian methods. Penalty approaches assume a smooth contact behaviour and the exact solution is recovered only for an infinite value of the penalty constant [11-15]. High penalty values lead to a set of stiff differential equations that produce ill-conditioned matrices and severe precision losses [16]. The displacement is the only primary variable in the formulation, leading to a relatively easy numerical implementation. Nevertheless, it requires a very small time step to capture the dynamic phenomena during the impact process yielding an expensive method. Additionally, the choice of the penalty factor by the user in order to obtain acceptable solutions that limit the unavoidable penetration between contacting bodies bears some arbitrariness. Lagrange multipliers methods guarantee the exact fulfilment of the constraints overcoming the ill-conditioning inconvenience of penalty methods at the expense of an increase in the size of the system of equations $[3,17]$. These methods can be related to nonlinear optimization problems, which permit the use of formulations with a solid mathematical background. A combination of the penalty and the Lagrange multipliers techniques leads to the so-called augmented Lagrangian methods [18-21]. The augmented Lagrangian method was proposed first by Hestenes [22] and Powell [23] to 
solve optimization problems with equality constraints. The augmented Lagrangian and the penalty methods both involve a penalty parameter. The role of the penalty factor in the augmented Lagrangian method is only to improve the convergence rate, whilst in the penalty method this factor directly influences the accuracy of the solution [24].

A proper selection of a time integration scheme is then required. Time integrators for nonsmooth dynamics are usually classified in two main groups, namely, event-driven and time-stepping time integrators. Event-driven schemes are based on the exact detection of the time of impact. They proved to be accurate but if a large number of events occur in a short period of time, they become inefficient. On the other hand, event-capturing time-stepping integrators are able to treat several impacts in a single time step, by formulating the contact constraints at the velocity level with an impact law. The Newton impact law relates the velocity after impact to the velocity before impact with a restitution coefficient. One of these methods, that was initially developed by Jean and Moreau [25,26], gives robust and efficient algorithms with a rigorous mathematical formulation. However, it leads to a first-order approximation of motion with high levels of numerical dissipation that compromise the accuracy of the computation of vibrations in flexible components and thus, a very small time step is required to get good results. Other drawback of this methodology is the constraint drift at the position level, because the constraints are imposed only at the velocity level. Currently, several nonsmooth contact dynamic algorithms are implemented in software packages, for example in LMGC90 [27], Siconos [28], and Oofelie [29].

In case of studying the dynamics of structures with a finite element spatial discretization, classical time integration schemes are the Newmark family methods [30], the Hilbert-Hughes-Taylor method (HHT) [31] and the generalized- $\alpha$ method [32]. These are implicit integrators that assume that the kinematic variables are smooth and consequently a second order precision is obtained $[13,33]$. These solvers can deal with stiff problems in structural dynamics with a broad frequency content [24]. However, when abrupt changes of the velocity appear in case of impact, these methods produce numerical solutions that show severe precision losses with a non physical behaviour and the generation of fictitious energy at the contact instant. To avoid this problem, time integration schemes that preserve the total energy of the system have been proposed in which the contact inequalities are transformed in equality constraints by using a slack variable [34,35]. The drawback of this kind of integrators is that they require the design of unconventional elements in the finite element library specifically adapted to the integrator.

A family of modified Newmark schemes specifically tailored for the time integration of flexible contact problems can be found in [36-38]. Although these schemes are shown to perform well, we are interested in contact prob- 
lems involving not only flexible but also rigid bodies and bilateral constraints which are usually found the modelling of multibody systems.

The nonsmooth generalized- $\alpha$ (NSGA) scheme was introduced by Brüls et al. [39] for modelling contact in multibody systems. It consists in splitting the involved fields into a (nonsmooth) impulsive contribution integrated with first-order accuracy, and a smooth contribution integrated with second-order accuracy by means of the generalized- $\alpha$ method. In this way, the smooth vibration phenomena are computed with small numerical dissipation, giving an accurate computation of the flexible dynamic response as shown in [40]. The NSGA also guarantees the exact satisfaction of the constraints both at velocity and at position levels, eliminating any drift problems. In other words, penetration is avoided both at position and velocity levels. Another important aspect of this methodology is that the final system of equations is solved at each time-step using a monolithic semi-smooth Newton method.

In a recent work, Cosimo et al. [41] presented a decoupled version of the nonsmooth generalized- $\alpha$ method. Two noticeable differences with respect to its predecessor [39] should be highlighted. On the one hand, the definition of the splitting is modified in order to ensure a decoupling of the three different subsets of equations that need to be solved at every time step. This feature improves considerably the robustness of the integrator for problems involving nonlinear bilateral constraints and flexible elements. On the other hand, the equations are formulated by using an augmented Lagrangian approach, which combines Lagrange multipliers and penalty terms. So far this methodology was only applied to frictionless contact and impact problems.

The objective of this work is to simulate the nonsmooth dynamics of multibody systems subject to impact and friction using the non-smooth generalized- $\alpha$ time integration scheme. The contact and friction kinematics selected for this study is a node-to-face element that can handle the large finite rotations originated by changes of orientation of the contact plane. This formulation is useful for treating rigid-rigid and rigid-flexible contact with friction, which appear in many types of mechanisms. We remark that flexible-flexible contact problems are generally handled using Mortar-based formulations as in $[36,42,43]$. The implementation of this approach in the context of the non-smooth generalized- $\alpha$ integration scheme is currently under development.

The article is organized as follows. Section 2 introduces the equations of motion. Section 3 gives a brief explanation of the nonsmooth generalized$\alpha$ time integrator for frictionless problems introduced in [41]. Section 4 presents the main contributions of this work, i.e., the formulation of the frictional contact element. In this section the internal force vectors and tangent matrices of the frictional contact element are presented. In section 5, several numerical examples are studied to evaluate the robustness and accuracy of the proposed frictional contact model. Finally, the main conclusions of the paper are summarized in section 6 . 


\section{Equations of motion}

After spatial semi-discretization, the equations of motion for a multibody system with unilateral and bilateral constraints are written in the following form at velocity level:

$$
\begin{aligned}
\dot{\boldsymbol{q}}^{+} & =\boldsymbol{v}^{+} & \\
\boldsymbol{M}(\boldsymbol{q}) \mathrm{d} \boldsymbol{v}-\boldsymbol{g}_{\boldsymbol{q}}^{T} \mathrm{~d} \boldsymbol{i} & =\boldsymbol{f}(\boldsymbol{q}, \boldsymbol{v}, t) \mathrm{d} t & \\
-\boldsymbol{g}_{\boldsymbol{q}}^{\overline{\mathcal{U}}} \boldsymbol{v}^{+} & =\boldsymbol{o} & \\
-\left(g_{N \boldsymbol{q}}^{j} \boldsymbol{v}^{+}+e_{N}^{j} g_{N \boldsymbol{q}}^{j} \boldsymbol{v}^{-}\right) & \in \partial \psi_{\mathbb{R}^{+}}\left(\mathrm{d} i_{N}^{j}\right), & \text { if } g_{N}^{j}(\boldsymbol{q}) \leq 0, \forall j \in \mathcal{U}(1 \mathrm{~d}) \\
-\left(\boldsymbol{g}_{T \boldsymbol{q}}^{j} \boldsymbol{v}^{+}+e_{T}^{j} \boldsymbol{g}_{T \boldsymbol{q}}^{j} \boldsymbol{v}^{-}\right) & \in \partial \psi_{C\left(\mathrm{~d} i_{N}^{j}\right)}\left(\mathrm{d} \boldsymbol{i}_{T}^{j}\right), & \text { if } g_{N}^{j}(\boldsymbol{q}) \leq 0, \forall j \in \mathcal{U}(1 \mathrm{e})
\end{aligned}
$$

where

- $\mathcal{U}$ denotes the set of indices of the unilateral constraints, $\overline{\mathcal{U}}$ is its complementarity set, i.e., the set of bilateral constraints and $\mathcal{C}=\mathcal{U} \cup \overline{\mathcal{U}}$ is the full set of constraints.

- $\boldsymbol{q}$ is the vector of coordinates.

- $\dot{\boldsymbol{q}}^{+}(t)=\lim _{\gamma \rightarrow t, \gamma>t} \dot{\boldsymbol{q}}(\gamma)$ and $\boldsymbol{v}^{+}(t)=\lim _{\gamma \rightarrow t, \gamma>t} \boldsymbol{v}(\gamma)$ are the right limits of the velocity. Meanwhile, $\boldsymbol{v}^{-}(t)=\lim _{\gamma \rightarrow t, \gamma<t} \boldsymbol{v}(\gamma)$ denotes the left limit of the velocity (i.e. the velocity before impact). These quantities are functions of bounded variation. The convention $\boldsymbol{v}(t)=$ $\boldsymbol{v}^{+}(t)$ and $\dot{\boldsymbol{q}}(t)=\dot{\boldsymbol{q}}^{+}(t)$ will be used in the remaining part of this work for conciseness in notation.

- $\mathrm{d} \boldsymbol{i}$ is the impulse measure of the contact reaction and the bilateral forces.

- $\mathrm{d} i_{N}$ and $\mathrm{d} \boldsymbol{i}_{T}$ are the impulse measures of the contact reaction forces in the normal and tangential directions, respectively.

- $\boldsymbol{g}$ is the combined set of bilateral and unilateral constraints, and $\boldsymbol{g}_{\boldsymbol{q}}(\boldsymbol{q})$ is the corresponding gradient.

- $g_{N \boldsymbol{q}}$ and $\boldsymbol{g}_{T \boldsymbol{q}}$ are the gradient matrices in the normal and tangential directions at the contact point, respectively.

- At impact, the velocities $\boldsymbol{v}$ and $\boldsymbol{v}^{-}$are related by the Newton impact law $g_{N \boldsymbol{q}}^{j} \boldsymbol{v}+e_{N}^{j} g_{N \boldsymbol{q}}^{j} \boldsymbol{v}^{-}=0$ in the normal direction, and $\boldsymbol{g}_{T \boldsymbol{q}}^{j} \boldsymbol{v}+$ $e_{T}^{j} \boldsymbol{g}_{T \boldsymbol{q}}^{j} \boldsymbol{v}^{-}=\boldsymbol{O}$ in the tangential direction.

- $e_{N}^{j} \in[0,1]$ and $e_{T}^{j} \in[-1,1]$ are the normal and the tangential coefficients of restitution at the contact point $j \in \mathcal{U}$, respectively. 
- $\psi_{\mathbb{R}^{+}}$is the indicator function of the real half line $\mathbb{R}^{+}$and $\partial \psi_{\mathbb{R}^{+}}$is the sub-differential of $\psi_{\mathbb{R}^{+}}$. Meanwhile, $\psi_{C\left(\mathrm{~d} i_{N}\right)}$ is the indicator function of a section of the Coulomb friction cone. Equations (1e) represent the frictional unilateral conditions.

- $\boldsymbol{f}(\boldsymbol{q}, \boldsymbol{v}, t)=\boldsymbol{f}^{e x t}(t)-\boldsymbol{f}^{\text {cin }}(\boldsymbol{q}, \boldsymbol{v})-\boldsymbol{f}^{\text {damp }}(\boldsymbol{q}, \boldsymbol{v})-\boldsymbol{f}^{\text {int }}(\boldsymbol{q})$ collects the external, complementary inertia, damping and internal forces.

- $\boldsymbol{M}(\boldsymbol{q})$ is the mass matrix which may, in general, depend on the coordinates.

- $\mathrm{d} \boldsymbol{v}$ is the differential measure associated with the velocity $\boldsymbol{v}$, assumed to be of bounded variation.

- $t$ is time, and $\mathrm{d} t$ is the corresponding standard Lebesgue measure.

\section{Nonsmooth generalized- $\alpha$ time integrator for fric- tionless problems}

The decoupled version of the nonsmooth generalized- $\alpha$ method presented in [41] is used to integrate the equations of motion. This integrator is characterized by a splitting of the involved fields into an impulsive contribution, which is integrated with first-order accuracy, and a smooth contribution, which is integrated with second order accuracy using a generalized- $\alpha$ method. Additionally, the equation of motion is reformulated such that the unilateral and bilateral constraints appear both at position and velocity levels. This procedure is inspired by the index-reduction proposed by Gear, Gupta and Leimkuhler for bilaterally constrained mechanical systems [44]. Various splitting strategies have been discuses in previous works $[39,41,45,46]$. The splitting proposed in [41] leads to a system of equations which can be arranged as three sub-problems which can be solved in a sequential decoupled manner without introducing any approximation. The final set of time discretized equations is next provided for the frictionless case. Further details and the analysis of the method can be found in [41]. The application of this integrator to problems with friction is studied in Section 4.

Let us consider a time step $\left(t_{n}, t_{n+1}\right]$ with the stepsize $h=t_{n+1}-t_{n}$. At time $t_{n+1}$, the splitting decomposes the physical displacement and velocity fields into smooth contributions $\tilde{\boldsymbol{q}}_{n+1}$ and $\tilde{\boldsymbol{v}}_{n+1}$ and nonsmooth contributions $\boldsymbol{U}_{n+1}$ and $\boldsymbol{W}_{n+1}$ according to

$$
\begin{aligned}
& \boldsymbol{q}_{n+1}=\tilde{\boldsymbol{q}}_{n+1}+\boldsymbol{U}_{n+1} \\
& \boldsymbol{v}_{n+1}=\tilde{\boldsymbol{v}}_{n+1}+\boldsymbol{W}_{n+1}
\end{aligned}
$$




\subsection{Computation of the smooth motion}

The smooth motion is defined by a modified form of the equations of motion at time step $n+1$ where the contributions of the unilateral constraints and associated reaction forces are ignored, i.e.,

$$
\begin{aligned}
& \boldsymbol{M}\left(\tilde{\boldsymbol{q}}_{n+1}\right) \dot{\tilde{\boldsymbol{v}}}_{n+1}-\boldsymbol{f}\left(\tilde{\boldsymbol{q}}_{n+1}, \tilde{\boldsymbol{v}}_{n+1}, t_{n+1}\right)- \\
& \boldsymbol{g}_{\tilde{\boldsymbol{q}}, n+1}^{\overline{\mathcal{U}}, T}\left(k_{s} \tilde{\boldsymbol{\lambda}}_{n+1}^{\overline{\mathcal{U}}}-p_{s} \boldsymbol{g}_{\tilde{\boldsymbol{q}}, n+1}^{\overline{\mathcal{U}}} \tilde{\boldsymbol{v}}_{n+1}\right)=\mathbf{O} \\
& -k_{s} \boldsymbol{g}_{\tilde{\boldsymbol{q}}, n+1}^{\overline{\mathcal{u}}} \tilde{\boldsymbol{v}}_{n+1}=\boldsymbol{O}
\end{aligned}
$$

with the notation $\boldsymbol{g}_{\tilde{\boldsymbol{q}}, n+1}^{T}=\boldsymbol{g}_{\tilde{\boldsymbol{q}}}^{T}\left(\tilde{\boldsymbol{q}}_{n+1}\right) ; \tilde{\boldsymbol{\lambda}}^{\overline{\mathcal{U}}}$ is a Lagrange multiplier associated with the bilateral constraint, $p_{s} \geq 0$ is the penalty parameter and $k_{s}>0$ is a scaling factor for the Lagrange multipliers. The scaling factor $k_{s}$ contributes to an improvement of the condition number of the iteration matrix, which yields in a better convergence rate. The advantage of using an augmented Lagrangian method is the presence of the penalty term with the penalty coefficient $p_{s}$ which adds convexity to the objective function and improves convergence of the Newton iteration far from the solution [24]. This factor does not influence the accuracy of the computed solution.

These equations are completed with the difference equations of the generalized- $\alpha$ scheme,

$$
\begin{aligned}
\tilde{\boldsymbol{q}}_{n+1} & =\boldsymbol{q}_{n}+h \boldsymbol{v}_{n}+h^{2}(0.5-\beta) \boldsymbol{a}_{n}+h^{2} \beta \boldsymbol{a}_{n+1} \\
\tilde{\boldsymbol{v}}_{n+1} & =\boldsymbol{v}_{n}+h(1-\gamma) \boldsymbol{a}_{n}+h \gamma \boldsymbol{a}_{n+1} \\
\left(1-\alpha_{m}\right) \boldsymbol{a}_{n+1}+\alpha_{m} \boldsymbol{a}_{n} & =\left(1-\alpha_{f}\right) \dot{\tilde{\boldsymbol{v}}}_{n+1}+\alpha_{f} \dot{\tilde{\boldsymbol{v}}}_{n}
\end{aligned}
$$

where $\boldsymbol{a}_{n+1}$ is a pseudo acceleration term that arises in the generalized- $\alpha$ integrator scheme [47]. The numerical coefficients $\gamma, \beta, \alpha_{m}$, and $\alpha_{f}$ can be chosen to achieve a desired level high-frequency dissipation, represented by spectral radius at infinity $\rho_{\infty} \in[0,1]$, while minimizing unwanted lowfrequency dissipation [32]:

$\alpha_{m}=\frac{2 \rho_{\infty}-1}{\rho_{\infty}+1}, \quad \alpha_{f}=\frac{\rho_{\infty}}{\rho_{\infty}+1}, \quad \gamma=0.5+\alpha_{f}-\alpha_{m}, \quad \beta=0.25(\gamma+0.5)^{2}$

Equations (3-4) involve only the smooth displacements $\tilde{\boldsymbol{q}}_{n+1}$ and velocities $\tilde{\boldsymbol{v}}_{n+1}$ and are thus decoupled from the variables $\boldsymbol{W}_{n+1}, \boldsymbol{U}_{n+1}, \boldsymbol{q}_{n+1}$, and $\boldsymbol{v}_{n+1}$. Thus, these five equations can be solved for the five variables $\tilde{\boldsymbol{q}}_{n+1}$, $\tilde{\boldsymbol{v}}_{n+1}, \tilde{\boldsymbol{\lambda}}_{n+1}^{\overline{\mathcal{U}}}, \dot{\boldsymbol{\boldsymbol { v }}}_{n+1}$ and $\boldsymbol{a}_{n+1}$ using a Newton-Raphson algorithm.

\subsection{Computation of the position correction}

After the computation of the smooth motion, the position correction $\boldsymbol{U}_{n+1}$ is computed in order to obtain a displacement $\boldsymbol{q}_{n+1}$ which satisfies the bilateral 
constraints $\boldsymbol{g}^{\overline{\mathcal{U}}}\left(\boldsymbol{q}_{n+1}\right)=\boldsymbol{O}$ and the non-penetration constraints $\boldsymbol{g}^{\mathcal{U}}\left(\boldsymbol{q}_{n+1}\right) \geq$ $\boldsymbol{0}$. An augmented Lagrangian approach as presented by Alart and Curnier [21] is adopted as described in [41]. At position level, the Lagrange multiplier of the unilateral and bilateral constraints is denoted by $\boldsymbol{\nu}$ and the augmented multiplier is defined as

$$
\boldsymbol{\xi}_{n+1}=k_{p} \boldsymbol{\nu}_{n+1}-p_{p} \boldsymbol{g}_{n+1}
$$

where $p_{p}>0$ is the penalty parameter, $k_{p}>0$ is the scale factor for the Lagrange multiplier $\boldsymbol{\nu}$ and $\boldsymbol{g}_{n+1}=\boldsymbol{g}\left(\boldsymbol{q}_{n+1}\right)$. The active set at position level $\mathcal{A} \equiv \mathcal{A}_{n+1}$ and its complement $\overline{\mathcal{A}} \equiv \overline{\mathcal{A}}_{n+1}$ are given by

$$
\begin{aligned}
& \mathcal{A}_{n+1}=\overline{\mathcal{U}} \cup\left\{j \in \mathcal{U}: \xi_{n+1}^{j} \geq 0\right\} \\
& \overline{\mathcal{A}}_{n+1}=\mathcal{C} \backslash \mathcal{A}_{n+1}
\end{aligned}
$$

The equations for the position correction are obtained as

$$
\begin{aligned}
\boldsymbol{M}\left(\tilde{\boldsymbol{q}}_{n+1}\right) \boldsymbol{U}_{n+1}-h^{2} \boldsymbol{f}_{n+1}^{p}-\boldsymbol{g}_{\boldsymbol{q}, n+1}^{\mathcal{A}, T} \boldsymbol{\xi}_{n+1}^{\mathcal{A}} & =\boldsymbol{0} \\
-k_{p} \boldsymbol{g}_{n+1}^{\mathcal{A}} & =\boldsymbol{0} \\
-\frac{k_{p}^{2}}{p_{p}} \boldsymbol{\nu}_{n+1}^{\mathcal{A}} & =\boldsymbol{O}
\end{aligned}
$$

where

$$
\boldsymbol{f}_{n+1}^{p}=\boldsymbol{f}\left(\boldsymbol{q}_{n+1}, \tilde{\boldsymbol{v}}_{n+1}, t_{n+1}\right)-\boldsymbol{f}\left(\tilde{\boldsymbol{q}}_{n+1}, \tilde{\boldsymbol{v}}_{n+1}, t_{n+1}\right)+\left(\boldsymbol{g}_{\boldsymbol{q}, n+1}^{\overline{\mathcal{U}}, T}-\boldsymbol{g}_{\tilde{\boldsymbol{q}}, n+1}^{\overline{\mathcal{u}}, T}\right) \tilde{\boldsymbol{\lambda}}_{n+1}^{\overline{\mathcal{U}}}
$$

This set of equations can be solved for the unknown variables $\boldsymbol{U}_{n+1}$ and $\boldsymbol{\nu}_{n+1}$ using a Newton semi-smooth method.

\subsection{Computation of the velocity jump}

After the computation of the displacement field, the velocity jump $\boldsymbol{W}_{n+1}$ is computed such that the velocity $\boldsymbol{v}_{n+1}$ satisfies the bilateral constraints $\boldsymbol{g}_{\boldsymbol{q}}^{\overline{\mathcal{u}}} \boldsymbol{v}_{n+1}=\boldsymbol{O}$ and the impact law $g_{\boldsymbol{q}, n+1}^{j} \boldsymbol{v}_{n+1}+e_{N}^{j} g_{\boldsymbol{q}, n}^{j} \boldsymbol{v}_{n}=0$ for all unilateral constraints $j \in \mathcal{U}$ that are active at position level, i.e., that satisfy $\xi_{n+1}^{j} \geq 0$. Again, an augmented Lagrangian approach is adopted as in [41], with the Lagrange multiplier of the unilateral and bilateral constraints at velocity level denoted by $\boldsymbol{\Lambda}$ and with the augmented multiplier defined by

$$
\boldsymbol{\sigma}_{n+1}=k_{v} \boldsymbol{\Lambda}_{n+1}-p_{v} \stackrel{\circ}{\operatorname{g}}_{n+1}
$$

where $p_{v}>0$ is the penalty parameter, $k_{v}>0$ is the scaling factor for the Lagrange multiplier $\boldsymbol{\Lambda}$, and $\stackrel{\circ}{\boldsymbol{g}}_{n+1}$ is a notation for the term of the impact law:

$$
\stackrel{\circ}{g}_{n+1}^{j}=g_{\boldsymbol{q}, n+1}^{j} \boldsymbol{v}_{n+1}+e_{N}^{j} g_{\boldsymbol{q}, n}^{j} \boldsymbol{v}_{n} \quad(=0)
$$


which applies for every $j \in \mathcal{C}$. The coefficients associated to bilateral constraints are trivially defined as $e_{N}^{j}=0 \forall j \in \mathcal{U}$. The active set $\mathcal{B} \equiv \mathcal{B}_{n+1}$ and its complement $\overline{\mathcal{B}} \equiv \overline{\mathcal{B}}_{n+1}$ are given by

$$
\begin{aligned}
& \mathcal{B}_{n+1}=\overline{\mathcal{U}} \cup\left\{j \in \mathcal{A}_{n+1}: \sigma_{n+1}^{j} \geq 0\right\} \\
& \overline{\mathcal{B}}_{n+1}=\mathcal{C} \backslash \mathcal{B}_{n+1}
\end{aligned}
$$

The equations for the velocity jump can be derived as

$$
\begin{aligned}
\boldsymbol{M}\left(\boldsymbol{q}_{n+1}\right) \boldsymbol{W}_{n+1}-h \boldsymbol{f}_{n+1}^{*}-\boldsymbol{g}_{\boldsymbol{q}, n+1}^{\mathcal{B}, T} \boldsymbol{\sigma}_{n+1}^{\mathcal{B}} & =\boldsymbol{O} \\
-k_{v} \stackrel{\boldsymbol{g}}{n+1}^{\mathcal{B}} & =\boldsymbol{O} \\
-\frac{k_{v}^{2}}{p_{v}} \boldsymbol{\Lambda}_{n+1}^{\overline{\mathcal{B}}} & =\boldsymbol{O}
\end{aligned}
$$

where

$$
\begin{aligned}
\boldsymbol{f}_{n+1}^{*}= & \boldsymbol{f}\left(\boldsymbol{q}_{n+1}, \boldsymbol{v}_{n+1}, t_{n+1}\right)-\boldsymbol{f}\left(\tilde{\boldsymbol{q}}_{n+1}, \tilde{\boldsymbol{v}}_{n+1}, t_{n+1}\right) \\
& +\left(\boldsymbol{g}_{\boldsymbol{q}, n+1}^{\overline{\mathcal{U}}, T}-\boldsymbol{g}_{\tilde{\boldsymbol{q}}, n+1}^{\bar{u}}\right) \tilde{\boldsymbol{\lambda}}_{n+1}^{\mathcal{U}}-\left(\boldsymbol{M}\left(\boldsymbol{q}_{n+1}\right)-\boldsymbol{M}\left(\tilde{\boldsymbol{q}}_{n+1}\right)\right) \dot{\boldsymbol{\boldsymbol { v }}}_{n+1}
\end{aligned}
$$

This set of equations can be solved for the unknown variables $\boldsymbol{W}_{n+1}$ and $\boldsymbol{\Lambda}_{n+1}$ using a Newton semi-smooth method.

\subsection{Global numerical procedure}

In the nonsmooth generalized- $\alpha$ scheme, the three sub-problems $(3,8,13)$ need to be solved at each time step for the smooth motion, the position correction and the velocity jump, with the computations organized in a sequential manner. The complete derivation of the equations of the different sub-problems and a detailed performance analysis of the resulting algorithm compared with [39] are described in detail in [41].

Let us introduce $\boldsymbol{r}^{s}, \boldsymbol{r}^{p}$ and $\boldsymbol{r}^{v}$ as the residuals of Eqs. (3), (8) and (13), respectively, as well as the vectors of independent corrections

$$
\Delta \boldsymbol{x}^{s}=\left\{\begin{array}{c}
\Delta \tilde{\boldsymbol{v}} \\
\Delta \tilde{\boldsymbol{\lambda}}^{\overline{\mathcal{U}}}
\end{array}\right\}, \quad \Delta \boldsymbol{x}^{p}=\left\{\begin{array}{c}
\Delta \boldsymbol{U} \\
\Delta \boldsymbol{\nu}^{\mathcal{A}} \\
\Delta \boldsymbol{\nu}^{\overline{\mathcal{A}}}
\end{array}\right\}, \quad \Delta \boldsymbol{x}^{v}=\left\{\begin{array}{c}
\Delta \boldsymbol{W} \\
\Delta \boldsymbol{\Lambda}^{\mathcal{B}} \\
\Delta \boldsymbol{\Lambda}^{\overline{\mathcal{B}}}
\end{array}\right\} .
$$

The correction equations for each sub-problem are obtained as

$$
\boldsymbol{S}_{t}^{i} \Delta \boldsymbol{x}^{i}=-\boldsymbol{r}^{i}, \quad \text { for } \quad i=s, p, v .
$$


The iteration matrices are respectively given by

$$
\begin{aligned}
\boldsymbol{S}_{t}^{s}= & {\left[\begin{array}{cc}
\boldsymbol{S}_{t}^{s^{*}} & -k_{s} \boldsymbol{g}_{\tilde{\boldsymbol{q}}, n+1}^{\overline{\mathcal{T}}, T} \\
-k_{s}\left(\boldsymbol{g}_{\tilde{\boldsymbol{q}}, n+1}^{\overline{\mathcal{U}}}+\frac{h \beta}{\gamma} \boldsymbol{G}^{\boldsymbol{s}}\right) & \boldsymbol{0}
\end{array}\right] } \\
\boldsymbol{S}_{t}^{p}= & {\left[\begin{array}{ccc}
\boldsymbol{S}_{t}^{p^{*}} & -k_{p} \boldsymbol{g}_{\boldsymbol{q}, n+1}^{\mathcal{A}, T} & \boldsymbol{0} \\
-k_{p} \boldsymbol{g}_{\boldsymbol{q}, n+1}^{\mathcal{A}} & \boldsymbol{0} & \boldsymbol{0} \\
\boldsymbol{0} & \boldsymbol{0} & -\frac{k_{p}^{2}}{p_{p}} \boldsymbol{I}^{\overline{\mathcal{A}}}
\end{array}\right] } \\
\boldsymbol{S}_{t}^{v}= & {\left[\begin{array}{ccc}
\boldsymbol{S}_{t}^{v^{*}} & -k_{v} \boldsymbol{g}_{\boldsymbol{q}, n+1}^{\mathcal{B}, T} & \boldsymbol{0} \\
-k_{v} \boldsymbol{g}_{\boldsymbol{q}, n+1}^{\mathcal{B}} & \boldsymbol{0} & \boldsymbol{0} \\
\boldsymbol{0} & \boldsymbol{0} & -\frac{k_{v}^{2}}{p_{v}} \boldsymbol{I}^{\overline{\mathcal{B}}}
\end{array}\right] }
\end{aligned}
$$

where $\boldsymbol{I}^{\overline{\mathcal{A}}}$ and $\boldsymbol{I}^{\overline{\mathcal{B}}}$ are identity matrices and

$$
\begin{aligned}
& \boldsymbol{S}_{t}^{s^{*}}=\frac{1-\alpha_{m}}{h\left(1-\alpha_{f}\right) \gamma} \boldsymbol{M}\left(\tilde{\boldsymbol{q}}_{n+1}\right)+\boldsymbol{C}_{t}+\frac{h \beta}{\gamma} \boldsymbol{K}_{t}^{s}, \\
& \boldsymbol{K}_{t}^{s}=\frac{\partial\left(\boldsymbol{M}\left(\tilde{\boldsymbol{q}}_{n+1}\right) \dot{\tilde{\boldsymbol{v}}}_{n+1}-\boldsymbol{g}_{\tilde{\boldsymbol{q}}, n+1}^{\overline{\mathcal{u}}, T}\left(k_{s} \tilde{\boldsymbol{\lambda}}_{n+1}^{\overline{\mathcal{u}}}-p_{s} \overline{\boldsymbol{g}}_{\tilde{\boldsymbol{q}}, n+1}^{\overline{\mathcal{v}}} \tilde{\boldsymbol{v}}_{n+1}\right)-\boldsymbol{f}\left(\tilde{\boldsymbol{q}}_{n+1}, \tilde{\boldsymbol{v}}_{n+1}, t_{n+1}\right)\right)}{\partial \tilde{\boldsymbol{q}}_{n+1}}, \\
& \boldsymbol{C}_{t}=p_{s} \boldsymbol{g}_{\tilde{\boldsymbol{q}}, n+1}^{\overline{\mathcal{U}}, T} \boldsymbol{g}_{\tilde{\boldsymbol{q}}, n+1}^{\overline{\mathcal{U}}}-\frac{\partial \boldsymbol{f}\left(\tilde{\boldsymbol{q}}_{n+1}, \tilde{\boldsymbol{v}}_{n+1}, t_{n+1}\right)}{\partial \tilde{\boldsymbol{v}}_{n+1}}, \quad \boldsymbol{G}^{s}=\frac{\partial\left(\boldsymbol{g}_{\tilde{\boldsymbol{q}}, n+1} \tilde{\boldsymbol{v}}_{n+1}\right)}{\partial \tilde{\boldsymbol{q}}_{n+1}}, \\
& \boldsymbol{S}_{t}^{p^{*}}=\boldsymbol{M}\left(\tilde{\boldsymbol{q}}_{n+1}\right)-\frac{\partial\left(\boldsymbol{g}_{\boldsymbol{q}, n+1}^{\mathcal{A}, T} \boldsymbol{\xi}_{n+1}^{\mathcal{A}}\right)}{\partial \boldsymbol{q}_{n+1}}-h^{2} \frac{\partial \boldsymbol{f}^{p}\left(\boldsymbol{q}_{n+1}, \tilde{\boldsymbol{q}}_{n+1}, \tilde{\boldsymbol{v}}_{n+1}, t_{n+1}\right)}{\partial \boldsymbol{q}_{n+1}}, \\
& \boldsymbol{S}_{t}^{v^{*}}=\boldsymbol{M}\left(\boldsymbol{q}_{n+1}\right)+p_{v} \boldsymbol{g}_{\boldsymbol{q}, n+1}^{\overline{\mathcal{B}}, T} \boldsymbol{g}_{\boldsymbol{q}, n+1}^{\overline{\mathcal{B}}}-h \frac{\partial \boldsymbol{f}^{*}\left(\boldsymbol{q}_{n+1}, \boldsymbol{v}_{n+1}, t_{n+1}\right)}{\partial \boldsymbol{v}_{n+1}}
\end{aligned}
$$

As mentioned before, the solution does not depend on the value of the parameters $k_{s}, k_{p}, k_{v}, p_{s}, p_{p}$ and $p_{v}$. Nevertheless, the convergence rate does depend on their values. In numerical computations, default values of the scaling and penalty parameters are selected according to the following relations given in $[48,49]$

$$
k_{s}=p_{s}=\frac{\bar{m}}{h}, \quad k_{p}=p_{p}=\bar{m}, \quad k_{v}=p_{v}=\bar{m},
$$

where $\bar{m}$ is a characteristic value of the mass of the problem. The steps involved by the integration algorithm are summarized in Algorithm 1. It should be observed that each sub-problem is solved with a Newton scheme. In practice this Newton scheme is complemented with a line-search strategy to improve the robustness of the resulting algorithm. 


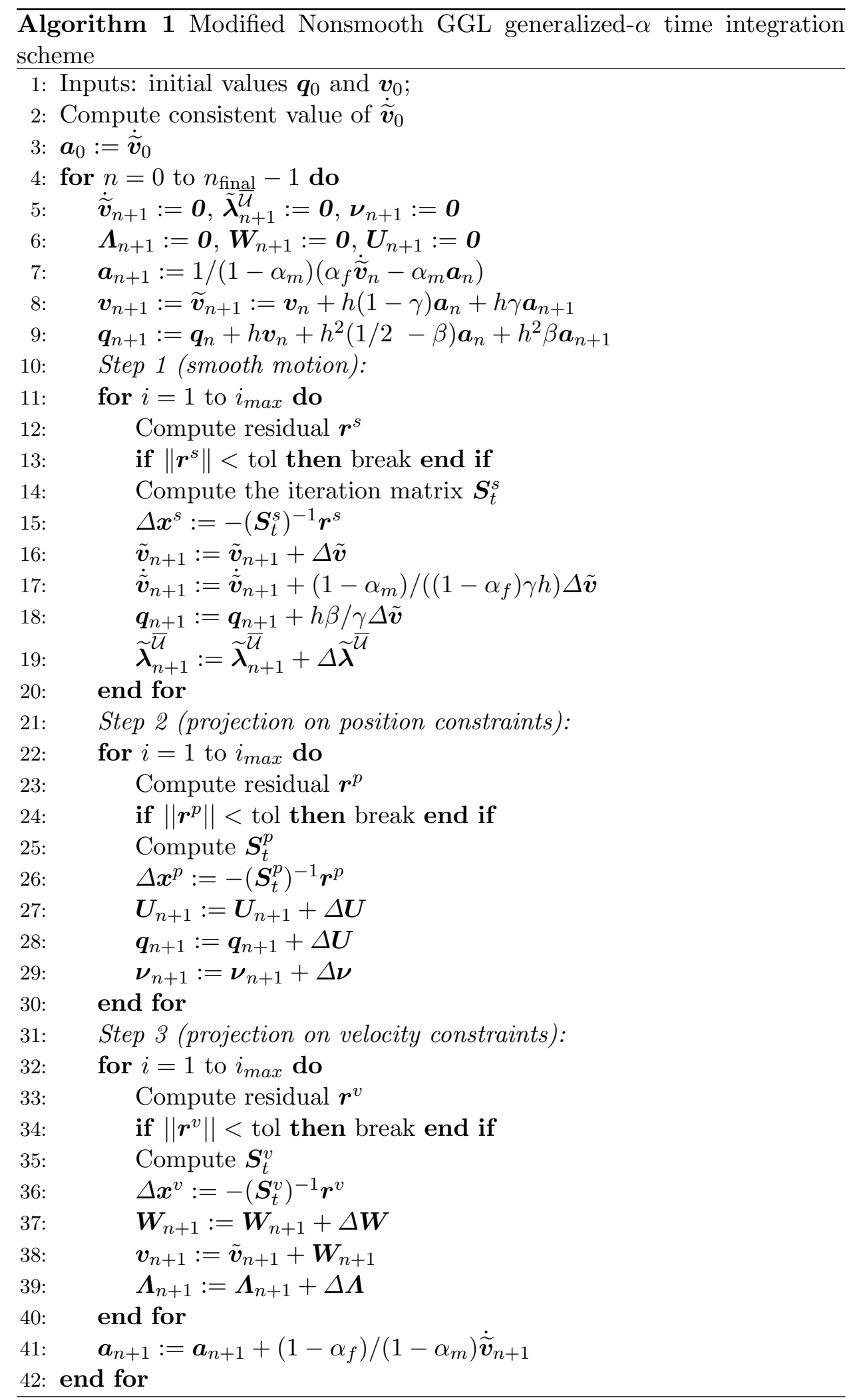




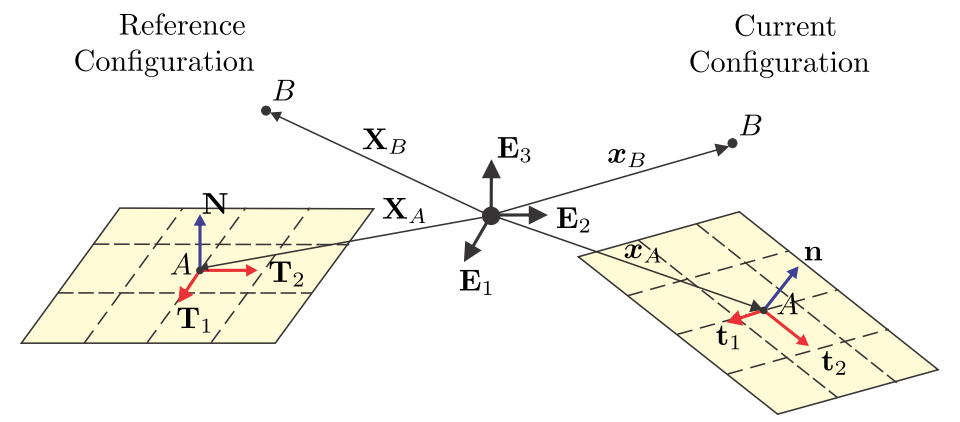

Figure 1: Node-to-face contact element.

\section{Frictional contact formulation}

The nonlinear Finite Element Method (FEM) is used in this work to analyze multibody systems composed of rigid and/or flexible elements and kinematic joints with different types of loading. By using the FEM approach, there is no distinction between the global coordinates of the rigid or flexible bodies. Therefore, the nonlinear effects are simply considered in the formulation with respect to a unique inertial frame [24].

By observing Eqs. $(8,13)$, the only terms that are related with the frictionless unilateral constraints can be compiled in the following global generalized internal force vectors at position and velocity levels, respectively,

$$
\begin{gathered}
\boldsymbol{F}_{G}^{p}\left(\boldsymbol{q}_{n+1}, \boldsymbol{\nu}_{n+1}\right)=\left\{\begin{array}{c}
-\boldsymbol{g}_{\boldsymbol{q}, n+1}^{\mathcal{A}, T} \boldsymbol{\xi}_{n+1}^{\mathcal{A}} \\
-k_{p} \boldsymbol{g}^{\mathcal{A}} \\
-\frac{k_{p}^{2}}{p_{p}} \boldsymbol{\nu}_{n+1}^{\overline{\mathcal{A}}}
\end{array}\right\} \\
\boldsymbol{F}_{G}^{v}\left(\boldsymbol{v}_{n+1}, \boldsymbol{\Lambda}_{n+1}\right)=\left\{\begin{array}{c}
-\boldsymbol{g}_{\boldsymbol{q}, T+1}^{\mathcal{B}, T} \boldsymbol{\sigma}_{n+1}^{\mathcal{B}} \\
-k_{v} \stackrel{\circ}{\mathcal{B}}^{\mathcal{B}} \\
-\frac{k_{v}^{2}}{p_{v}} \boldsymbol{\Lambda}_{n+1}^{\mathcal{B}}
\end{array}\right\}
\end{gathered}
$$

From Eqs. $(18,19)$ the global tangent matrix at position and velocity levels are

$$
\begin{aligned}
\boldsymbol{K}_{G}^{p}= & {\left[\begin{array}{ccc}
-\boldsymbol{g}_{\boldsymbol{q} \boldsymbol{q}, n+1}^{\mathcal{A}, T} \boldsymbol{\xi}_{n+1}^{A} & -k_{p} \boldsymbol{g}_{\boldsymbol{q}, n+1}^{\mathcal{A}, T} & \boldsymbol{0} \\
-k_{p} \boldsymbol{g}_{\boldsymbol{q}, n+1}^{\mathcal{A}} & \boldsymbol{0} & \boldsymbol{0} \\
\boldsymbol{O} & \boldsymbol{0} & -\frac{k_{p}^{2}}{p_{p}} \boldsymbol{I}^{\overline{\mathcal{A}}}
\end{array}\right] } \\
\boldsymbol{K}_{G}^{v}= & {\left[\begin{array}{ccc}
\boldsymbol{0} & -k_{v} \boldsymbol{g}_{\boldsymbol{q}, n+1}^{\mathcal{B}, T} & \boldsymbol{O} \\
-k_{v} \boldsymbol{g}_{\boldsymbol{q}, n+1}^{\mathcal{B}} & \boldsymbol{O} & \boldsymbol{O} \\
\boldsymbol{O} & \boldsymbol{O} & -\frac{k_{v}^{2}}{p_{v}} \boldsymbol{I}^{\overline{\mathcal{B}}}
\end{array}\right] }
\end{aligned}
$$

In a similar way, we consider that the frictional contact element does not contribute to the smooth motion and only participates to the position 
and velocity equations through vectors $\boldsymbol{F}_{G}^{p}\left(\boldsymbol{q}_{n+1}, \boldsymbol{\nu}_{n+1}\right)$ and $\boldsymbol{F}_{G}^{v}\left(\boldsymbol{v}_{n+1}, \boldsymbol{\Lambda}_{n+1}\right)$ that will be detailed below.

The proposed node-to-face frictional contact element is formulated in the framework of finite displacements and rotations. It is composed of a master planar rigid face in space with reference node $A$ in body $\mathcal{B}^{1}$, and a single node $B$ belonging to body $\mathcal{B}^{2}$, see Fig. 1 . For conciseness, this study focuses on the contact of a node with a planar external surface of a body.

The coordinates of the contact element $\boldsymbol{q}$ include the positions $\boldsymbol{x}_{A}$ and $\boldsymbol{x}_{B}$ of nodes $A$ and $B$, respectively, and the rotation operator $\boldsymbol{R}_{A}$ of node $A$. For the treatment of the rotation operator, we follow a similar formulation and solution strategy as described in [50]. The rotational velocity of node $A$ is thus represented in the velocity vector $\boldsymbol{v}$ by the three components of the angular velocity vector $\Omega_{A}$ in the local frame of node $A$. For this rotation variable, Eq. (1a) is replaced by

$$
\dot{\boldsymbol{R}}_{A}=\boldsymbol{R}_{A} \widetilde{\Omega}_{A}
$$

where the operator $\widetilde{\boldsymbol{u}}: \mathbb{R}^{3} \rightarrow \mathbb{R}^{3} \otimes \mathbb{R}^{3}$ returns a $3 \times 3$ skew-symmetric matrix such that $\boldsymbol{u} \times \boldsymbol{v}=\widetilde{\boldsymbol{u}} \boldsymbol{v}=-\boldsymbol{v} \times \boldsymbol{u}, \forall \boldsymbol{u}, \boldsymbol{v} \in \mathbb{R}^{3}$. Let us observe that, in the scope of this section 4, the "tilde" operator has a different meaning than in sections 2 and 3. Following the principle of the Lie group solver [50], the rotation at time step $n+1$ is represented as an increment vector $\boldsymbol{\Psi}_{A, n+1} \in \mathbb{R}^{3}$ with respect to the rotation at time step $n$ as

$$
\boldsymbol{R}_{A, n+1}=\boldsymbol{R}_{A, n} \exp \left(\widetilde{\boldsymbol{\Psi}}_{A, n+1}\right)
$$

which involves the exponential map on the rotation group. The nonsmooth generalized- $\alpha$ time integration formulae are then expressed in terms of the unknowns $\boldsymbol{\Psi}_{A, n+1}$ and $\boldsymbol{\Omega}_{A, n+1}$ at position and velocity levels, respectively.

The inertial frame is defined by a set of orthonormal vectors $\boldsymbol{E}_{1}, \boldsymbol{E}_{2}$ and $\boldsymbol{E}_{3}$, see Fig 1. During motion, node $B$ undergoes a displacement, meanwhile the rigid plane rotates and translates. The normal to the contact surface in the reference configuration is given by $\boldsymbol{N}$, meanwhile the vectors $\boldsymbol{T}_{1}$ and $\boldsymbol{T}_{2}$ are tangent to the contact surface. The positions of nodes $A$ and $B$ in the reference configuration are $\boldsymbol{X}_{A}$ and $\boldsymbol{X}_{B}$, respectively, and the positions of nodes $A$ and $B$ in the current configuration are given by vectors $\boldsymbol{x}_{A}$ and $\boldsymbol{x}_{B}$, respectively. During motion, the new orientations of $\boldsymbol{N}, \boldsymbol{T}_{1}$ and $\boldsymbol{T}_{2}$ at the current configuration, are denoted by vectors $\boldsymbol{n}, \boldsymbol{t}_{1}$ and $\boldsymbol{t}_{2}$, respectively:

$$
\begin{aligned}
\boldsymbol{n}_{n+1} & =\boldsymbol{R}_{A, n+1} \boldsymbol{N} \\
\boldsymbol{t}_{1, n+1} & =\boldsymbol{R}_{A, n+1} \boldsymbol{T}_{1} \\
\boldsymbol{t}_{2, n+1} & =\boldsymbol{R}_{A, n+1} \boldsymbol{T}_{2}
\end{aligned}
$$

The normal gap between nodes $A$ and $B$ in the current configuration is

$$
g_{N, n+1}=\boldsymbol{N}^{T} \boldsymbol{R}_{A, n+1}^{T}\left(\boldsymbol{x}_{B, n+1}-\boldsymbol{x}_{A, n+1}\right)
$$


The normal gap is used to evaluate if the interacting bodies come in contact with each other (a zero gap indicates contact). From a geometrical point of view, the normal gap $g_{N}$ represents the shortest distance from node $A$ to the contact surface at the current configuration. Thus, $g_{N}>0$ implies that the bodies are not in contact. In order to account for friction when the bodies are in contact, i.e if $g_{N}=0$ in the time interval $\left(t_{n}, t_{n+1}\right)$, the incremental tangential movement with components $g_{T 1, n+1}, g_{T 2, n+1}$ referred to the material frame $\boldsymbol{T}_{1}, \boldsymbol{T}_{2}$, is computed as follows

$$
\begin{aligned}
& g_{T 1, n+1}=\boldsymbol{T}_{1}^{T}\left[\boldsymbol{R}_{A, n+1}^{T}\left(\boldsymbol{x}_{B, n+1}-\boldsymbol{x}_{A, n+1}\right)-\boldsymbol{R}_{A, n}^{T}\left(\boldsymbol{x}_{B, n}-\boldsymbol{x}_{A, n}\right)\right] \\
& g_{T 2, n+1}=\boldsymbol{T}_{2}^{T}\left[\boldsymbol{R}_{A, n+1}^{T}\left(\boldsymbol{x}_{B, n+1}-\boldsymbol{x}_{A, n+1}\right)-\boldsymbol{R}_{A, n}^{T}\left(\boldsymbol{x}_{B, n}-\boldsymbol{x}_{A, n}\right)\right]
\end{aligned}
$$

Finally, the generalized gap vector is defined as

$$
\begin{aligned}
\boldsymbol{g}_{n+1}= & \left\{\begin{array}{l}
g_{N, n+1} \\
\boldsymbol{g}_{T, n+1}
\end{array}\right\}= \\
& \left\{\begin{array}{c}
\boldsymbol{N}^{T} \boldsymbol{R}_{A, n+1}^{T}\left(\boldsymbol{x}_{B, n+1}-\boldsymbol{x}_{A, n+1}\right) \\
\boldsymbol{T}_{1}^{T}\left[\boldsymbol{R}_{A, n+1}^{T}\left(\boldsymbol{x}_{B, n+1}-\boldsymbol{x}_{A, n+1}\right)-\boldsymbol{R}_{A, n}^{T}\left(\boldsymbol{x}_{B, n}-\boldsymbol{x}_{A, n}\right)\right. \\
\boldsymbol{T}_{2}^{T}\left[\boldsymbol{R}_{A, n+1}^{T}\left(\boldsymbol{x}_{B, n+1}-\boldsymbol{x}_{A, n+1}\right)-\boldsymbol{R}_{A, n}^{T}\left(\boldsymbol{x}_{B, n}-\boldsymbol{x}_{A, n}\right)\right.
\end{array}\right]
\end{aligned}
$$

where the first component is the normal gap, while the second and the third components are the tangential displacements. In what follows, we will adopt the subscripts $T$ and $N$ for denoting the components of a given quantity in the tangential and in the normal directions, respectively.

\subsection{Frictional contact problem at position level}

The modelling of friction represents an additional complexity with respect to the frictionless problem, as a consequence of the non-conservative character of the friction forces. The most popular model is given by the Coulomb friction law. In this model, the bodies in contact can be in two different states: stick or slip. In stick, there is no relative displacement between the bodies, meanwhile in slip the bodies slide on each other. At position level, the friction law should be expressed in terms of the main unknowns $\boldsymbol{U}_{n+1}$ and $\boldsymbol{\nu}_{n+1}$ (with the splitting $\boldsymbol{q}_{n+1}=\widetilde{\boldsymbol{q}}_{n+1}+\boldsymbol{U}_{n+1}$ ). The restrictions of gap, contact, stick or slip, described before, are represented by the following frictional contact conditions,

$$
\begin{aligned}
& g_{N, n+1} \geq 0, \quad \nu_{N, n+1} \geq 0, \quad \quad g_{N, n+1} \nu_{N, n+1}=0 \\
& \left\|\boldsymbol{g}_{T, n+1}\right\| \geq 0, \quad\left\|\boldsymbol{\nu}_{T, n+1}\right\| \leq \mu \nu_{N, n+1}, \\
& \quad\left\|\boldsymbol{g}_{T, n+1}\right\|\left(\left\|\boldsymbol{\nu}_{T, n+1}\right\|-\mu \nu_{N, n+1}\right)=0 \\
& \boldsymbol{g}_{T, n+1}=-\left\|\boldsymbol{g}_{T, n+1}\right\| \frac{\boldsymbol{\nu}_{T, n+1}}{\left\|\boldsymbol{\nu}_{T, n+1}\right\|}
\end{aligned}
$$


where $\nu_{N}$ and $\boldsymbol{\nu}_{T}$ are the normal and the tangential Lagrange multipliers at position level in the normal and tangential directions, respectively, and collinearity between the tangential displacement and the tangential contact force is assumed with the tangential force opposed to motion. The first condition in Eq. (34a) indicates the impenetrability restriction; the second one is the non-traction condition (only compression is allowed) and the third one is the complementarity equation. The second set of restrictions, Eq. (34b), represents the conditions for friction. The first inequality, that is always satisfied, states that the tangential displacement is positive or zero; the second one establishes that the maximum value of the density of the tangential contact force is $\mu \nu_{N, n+1}$, where $\mu$ is the friction coefficient; the third one is the complementary equation, which indicates that $\left\|\boldsymbol{g}_{T, n+1}\right\|$ and $\left\|\boldsymbol{\nu}_{T, n+1}\right\|-\mu \nu_{N, n+1}$ cannot be simultaneously different from zero. Hence, when $\left\|\boldsymbol{\nu}_{T, n+1}\right\|<\mu \nu_{N, n+1}$ and $\left\|\boldsymbol{g}_{T, n+1}\right\|=0$ the contact status is in stick, and when $\left\|\boldsymbol{g}_{T, n+1}\right\| \neq 0$, the body slips and the tangential force is equal to $\left\|\nu_{T, n+1}\right\|=\mu \nu_{N, n+1}$.

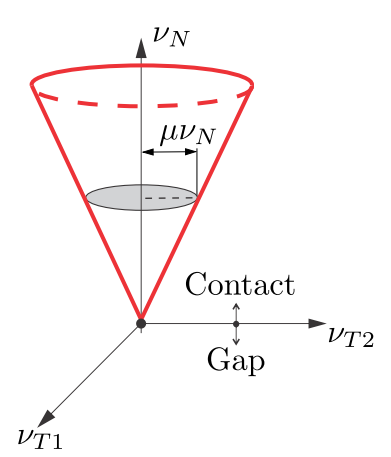

(a) Coulomb isotropic friction cone

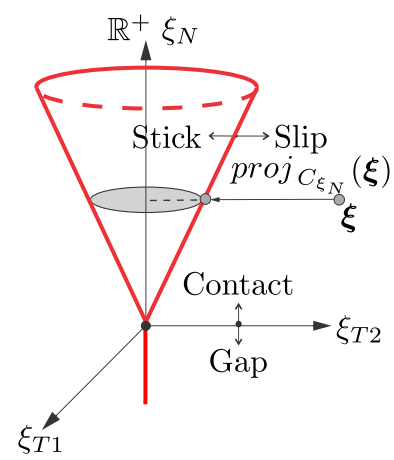

(b) Extension of the augmented Coulomb isotropic friction cone

Figure 2: Three dimensional representation of the Coulomb friction cone.

It is important to remark that, in the proposed framework, it is mandatory to impose the Coulomb friction constraint at position level. In order to understand this, recall that the time integration scheme is characterized by three decoupled sub-problems, and that the correction at position level is blind to any correction done at velocity level for the same time step where the terms are independent. Therefore, if the friction constraints are imposed only at velocity level some non-physical behaviour can be observed at position level, as shown in more detail in the application example in 5.1.

The set of constraints given in Eq. (34) could have been expressed in the form of an inclusion as in Eq. (1). In this work, from a convex analysis as in Alart and Curnier [21], the frictional contact problem is solved by transforming the normal and tangential inclusions into equivalent equations 
by using the proximal point algorithm [51]. For the sake of conciseness, in what follows, we only work with the part of the augmented Lagrangian corresponding to the unilateral constraints of a specific contact element. A similar approach was used in [41]. The adopted form of the augmented Lagrangian approach can be derived from the following function expressed in terms of the variables $\boldsymbol{q}_{n+1}$ and $\boldsymbol{\nu}_{n+1}$ defined in the global frame of reference.

$$
\begin{aligned}
\mathcal{L}^{p}\left(\boldsymbol{q}_{n+1}, \boldsymbol{\nu}_{n+1}\right) & =-k_{p} g_{N, n+1} \nu_{N, n+1}+\frac{p_{p}}{2}\left(g_{N, n+1}\right)^{2}- \\
& \frac{1}{2 p_{p}} \operatorname{dist}^{2}\left[k_{p} \nu_{N, n+1}-p_{p} g_{N, n+1}, \mathbb{R}^{+}\right]-k_{p} \boldsymbol{g}_{T, n+1} \cdot \boldsymbol{\nu}_{T, n+1}+ \\
& \frac{p_{p}}{2}\left\|\boldsymbol{g}_{T, n+1}\right\|^{2}-\frac{1}{2 p_{p}} \operatorname{dist}^{2}\left[k_{p} \boldsymbol{\nu}_{T, n+1}-p_{p} \boldsymbol{g}_{T, n+1}, C_{\xi_{N}}\right]
\end{aligned}
$$

where it is understood that the gap function depends on $\boldsymbol{q}$, though this is not explicitly mentioned for the sake of conciseness.

In order to facilitate the presentation of the problem, the augmented Lagrangian is split in two terms: the normal and the tangential contributions

$$
\mathcal{L}^{p}\left(\boldsymbol{q}_{n+1}, \boldsymbol{\nu}_{n+1}\right)=\mathcal{L}_{N}^{p}\left(\boldsymbol{q}_{n+1}, \nu_{N, n+1}\right)+\mathcal{L}_{T}^{p}\left(\boldsymbol{q}_{n+1}, \boldsymbol{\nu}_{T, n+1}\right)
$$

where the augmented Lagrangian in the normal direction is

$$
\begin{array}{r}
\mathcal{L}_{N}^{p}\left(\boldsymbol{q}_{n+1}, \nu_{N, n+1}\right)=-k_{p} g_{N, n+1} \nu_{N, n+1}+\frac{p_{p}}{2}\left(g_{N, n+1}\right)^{2}- \\
\frac{1}{2 p_{p}} \operatorname{dist}^{2}\left[\xi_{N, n+1}, \mathbb{R}^{+}\right]
\end{array}
$$

while in the tangential direction it is given by

$$
\begin{array}{r}
\mathcal{L}_{T}^{p}\left(\boldsymbol{q}_{n+1}, \boldsymbol{\nu}_{T, n+1}\right)=-k_{p} \boldsymbol{g}_{T, n+1} \cdot \boldsymbol{\nu}_{T, n+1}+\frac{p_{p}}{2}\left\|\boldsymbol{g}_{T, n+1}\right\|^{2}- \\
\frac{1}{2 p_{p}} \operatorname{dist}^{2}\left[\boldsymbol{\xi}_{T, n+1}, C_{\xi_{N}}\right]
\end{array}
$$

The function $\operatorname{dist}(\boldsymbol{z}, C)$ represents the distance between a point $\boldsymbol{z} \in \mathbb{R}^{n}$ and a convex set $C$, see

citeLeineBook2008. The normal part of the Lagrangian is based on the definition of the distance to the set $\mathbb{R}^{+}$:

$$
\operatorname{dist}\left(\xi_{N}, \mathbb{R}^{+}\right)=\left\{\begin{array}{cll}
-\xi_{N} & \text { if } \quad \xi_{N}<0 & \text { Gap } \\
0 & \text { if } \quad \xi_{N} \geq 0 & \text { Contact }
\end{array}\right.
$$

The Coulomb isotropic friction law, in 3D problems, is represented by a cone as shown in Fig. 2(a). A section of the Coulomb cone of radius $\mu \nu_{N}$ is defined by

$$
C\left(\nu_{N}\right)=\left\{\boldsymbol{\nu}_{T} \text { s.t. }\left\|\boldsymbol{\nu}_{T}\right\| \leq \mu \nu_{N}\right\}
$$


which represents the set of admissible tangential friction forces. Then, the extended augmented cone $C_{\xi_{N}}$ is the convex set defined by extension of the friction cone to the half line $\mathbb{R}^{-}\left(\xi_{N}\right)$, i.e., the set of negative values of the normal augmented multiplier, see Fig.2 (b). The tangential part of the Lagrangian is based on the definition of the distance to the extended augmented friction cone:

$$
\operatorname{dist}\left(\boldsymbol{\xi}_{T} ; C_{\xi_{N}}\right)=\left\{\begin{array}{cccccc}
\boldsymbol{\xi}_{T} & \text { if } & \xi_{N} & < & 0 & \text { Gap } \\
\boldsymbol{\xi}_{T}-\mu \xi_{N} \boldsymbol{\tau}_{p} & \text { if } & \left\|\boldsymbol{\xi}_{T}\right\| & \geq \mu \xi_{N} & \text { Slip } \\
\boldsymbol{0} & \text { if } & \left\|\boldsymbol{\xi}_{T}\right\| & <\mu \xi_{N} & \text { Stick }
\end{array}\right.
$$

where $\boldsymbol{\tau}_{p}=\boldsymbol{\xi}_{T} /\left\|\boldsymbol{\xi}_{T}\right\|$ is a unit vector pointing in the direction of the tangential contact force.

\subsubsection{Force vector in the normal direction}

The generalized internal force vector in the normal direction is obtained by taking variations of Eq. (37),

$$
\delta \mathcal{L}_{N}^{p}\left(\boldsymbol{q}, \nu_{N}\right)=-k_{p} \delta \nu_{N} g_{N}-\xi_{N} \delta g_{N}-\frac{1}{p_{p}} \delta\left[\frac{1}{2} \operatorname{dist}^{2}\left(\xi_{N}, \mathbb{R}^{+}\right)\right]
$$

where $\xi_{N}=k_{p} \nu_{N}-p_{p} g_{N}$ is the augmented multiplier in the normal direction. In this equation, the subindex $n+1$ is omitted for conciseness. This notation is adopted for the rest of the work. The variation of $(1 / 2) \operatorname{dist}^{2}\left(\xi_{N}, \mathbb{R}^{+}\right)$in Eq. (42) gives

$$
\delta\left[\frac{1}{2} \operatorname{dist}^{2}\left(\xi_{N}, \mathbb{R}^{+}\right)\right]=\left\{\begin{array}{cccc}
\xi_{N} \delta \xi_{N} & \text { if } & \xi_{N}<0 & \text { Gap } \\
0 & \text { if } & \xi_{N} \geq 0 & \text { Contact }
\end{array}\right.
$$

The virtual variation of the normal gap in Eq. (31), is written as follows

$$
\delta g_{N}=\boldsymbol{n}^{T}\left(\delta \boldsymbol{x}_{B}-\delta \boldsymbol{x}_{A}\right)+\boldsymbol{N}^{T} \widehat{\boldsymbol{R}_{A}^{T}\left(\boldsymbol{x}_{B}-\boldsymbol{x}_{A}\right)} \delta \boldsymbol{\Theta}_{A}
$$

where the variation of the normal $\delta \boldsymbol{n}=-\boldsymbol{R}_{A} \tilde{\boldsymbol{N}} \delta \boldsymbol{\Theta}_{A}$ was used. Here, $\delta \boldsymbol{\Theta}_{A}$ is the variation of the incremental material rotations at node $A$ such that $\delta \boldsymbol{R}_{A}=\boldsymbol{R}_{A} \widetilde{\delta \boldsymbol{\Theta}_{A}}$, [24]. In order to obtain a compact expression for the normal gap variation, Eq. (44) can be written as follows

$$
\delta g_{N}=g_{N \boldsymbol{q}} \delta \boldsymbol{q}
$$

where the gradient matrix in the normal direction is

$$
g_{N \boldsymbol{q}}=\left[\begin{array}{lll}
-\boldsymbol{n}^{T} & \boldsymbol{N}^{T} \widetilde{\boldsymbol{d}_{A B}} & \boldsymbol{n}^{T}
\end{array}\right]
$$

with $\boldsymbol{d}_{A B}=\boldsymbol{R}_{A}^{T}\left(\boldsymbol{x}_{B}-\boldsymbol{x}_{A}\right) ; \delta \boldsymbol{q}$ is the variation of the nodal coordinates for a contact element with nodes $A, B$, where the positions and rotations are ordered in the following way

$$
\delta \boldsymbol{q}=\left[\delta \boldsymbol{x}_{A}^{T} \delta \boldsymbol{\Theta}_{A}^{T} \delta \boldsymbol{x}_{B}^{T}\right]^{T}
$$


Replacing Eqs. (43, 45, 46) into Eq. (42), the generalized internal forces vector of the contact element $\boldsymbol{F}_{N}^{p}$ can then be readily identified as conjugated to the variation of generalized coordinates of the element $\boldsymbol{\Phi}=\left[\begin{array}{ll}\boldsymbol{q}^{T} & \nu_{N} \\ \boldsymbol{\nu}_{T}^{T}\end{array}\right]^{T}$ as follows,

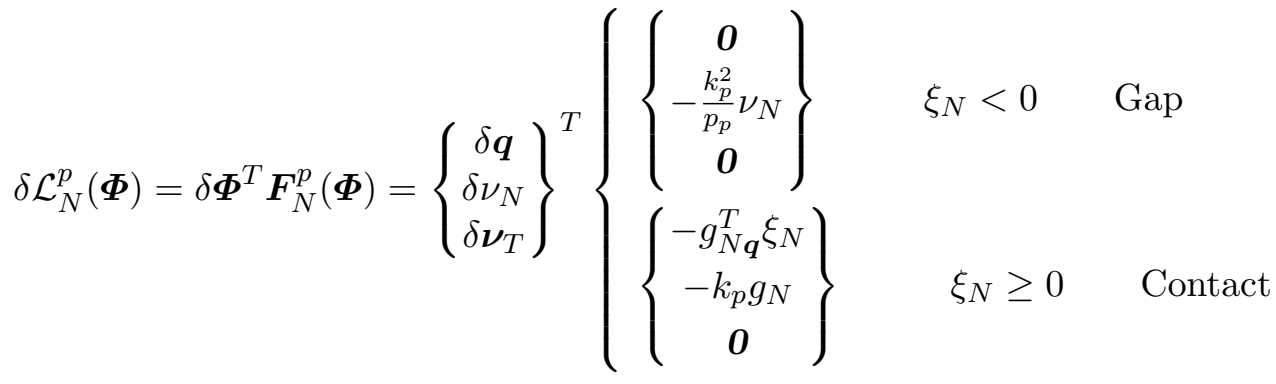

\subsubsection{Force vector in the tangential direction}

The generalized internal force vector in the tangential direction is computed in a similar way. By taking variations of Eq. (38) the following equation is obtained

$$
\delta \mathcal{L}_{T}^{p}\left(\boldsymbol{q}, \boldsymbol{\nu}_{T}\right)=-k_{p} \delta \boldsymbol{\nu}_{T} \cdot \boldsymbol{g}_{T}-\boldsymbol{\xi}_{T} \cdot \delta \boldsymbol{g}_{T}-\frac{1}{p_{p}} \delta\left[\frac{1}{2} \operatorname{dist}^{2}\left(\boldsymbol{\xi}_{T}, C_{\xi_{N}}\right)\right]
$$

The variation of $(1 / 2) \operatorname{dist}^{2}\left(\boldsymbol{\xi}_{T} ; C_{\xi_{N}}\right)$ is

$\delta\left[\frac{1}{2} \operatorname{dist}^{2}\left(\boldsymbol{\xi}_{T} ; C_{\xi_{N}}\right)\right]=\left\{\begin{array}{clccc}\boldsymbol{\xi}_{T} \cdot \delta \boldsymbol{\xi}_{T} & \text { if } \xi_{N} & < & 0 & \text { Gap } \\ \left(\left\|\boldsymbol{\xi}_{T}\right\|-\mu \xi_{N}\right) \boldsymbol{\tau}_{p} \cdot \delta \boldsymbol{\xi}_{T} & \text { if }\left\|\boldsymbol{\xi}_{T}\right\| & \geq \mu \xi_{N} & \text { Slip (50) } \\ 0 & \text { if }\left\|\boldsymbol{\xi}_{T}\right\| & <\mu \xi_{N} & \text { Stick }\end{array}\right.$

where $\boldsymbol{\tau}_{p}=\boldsymbol{\xi}_{T} /\left\|\boldsymbol{\xi}_{T}\right\|$ is the direction of the tangential contact force at position level. Then, the virtual variation of the tangential displacement $\boldsymbol{g}_{T}$ (Eq. (32)) is given by

$$
\delta \boldsymbol{g}_{T}=\boldsymbol{g}_{T \boldsymbol{q}} \delta \boldsymbol{q}
$$

with the gradient matrix in the tangential direction defined as

$$
\boldsymbol{g}_{T \boldsymbol{q}}=\left[\begin{array}{ccc}
-\boldsymbol{t}_{1}^{T} & \boldsymbol{T}_{1}^{T} \widetilde{\boldsymbol{d}_{A B}} & \boldsymbol{t}_{1}^{T} \\
-\boldsymbol{t}_{2}^{T} & \boldsymbol{T}_{2}^{T} \widetilde{\boldsymbol{d}_{A B}} & \boldsymbol{t}_{2}^{T}
\end{array}\right]
$$

Finally, replacing Eqs. $(50,51)$ in Eq. (49), the generalized internal forces vector of the contact element $\boldsymbol{F}_{T}^{p}$ for the status of gap, slip or stick, is given 
by

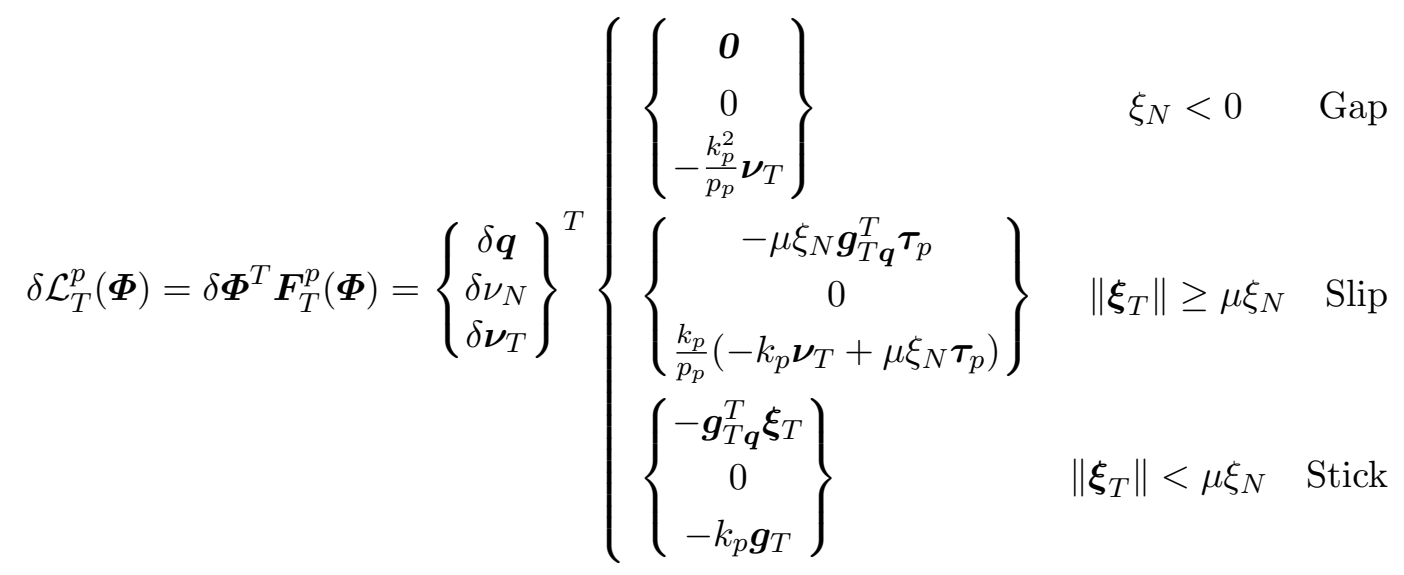

\subsubsection{Force vector and Hessian matrix at position level}

The final expression of the internal contact force vector for the different contact status are obtained by adding the normal and tangential contributions of the internal forces vector from Eqs. $(48,53)$.

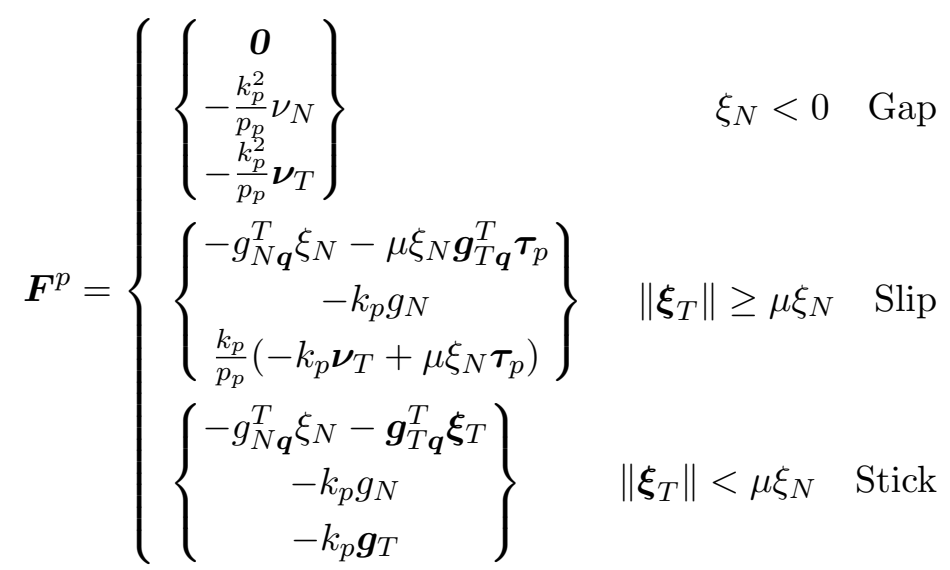

The linearization of the internal force vector gives the contact Hessian matrix. The contributions to the Hessian matrix for the different contact 
conditions are expressed as follows:

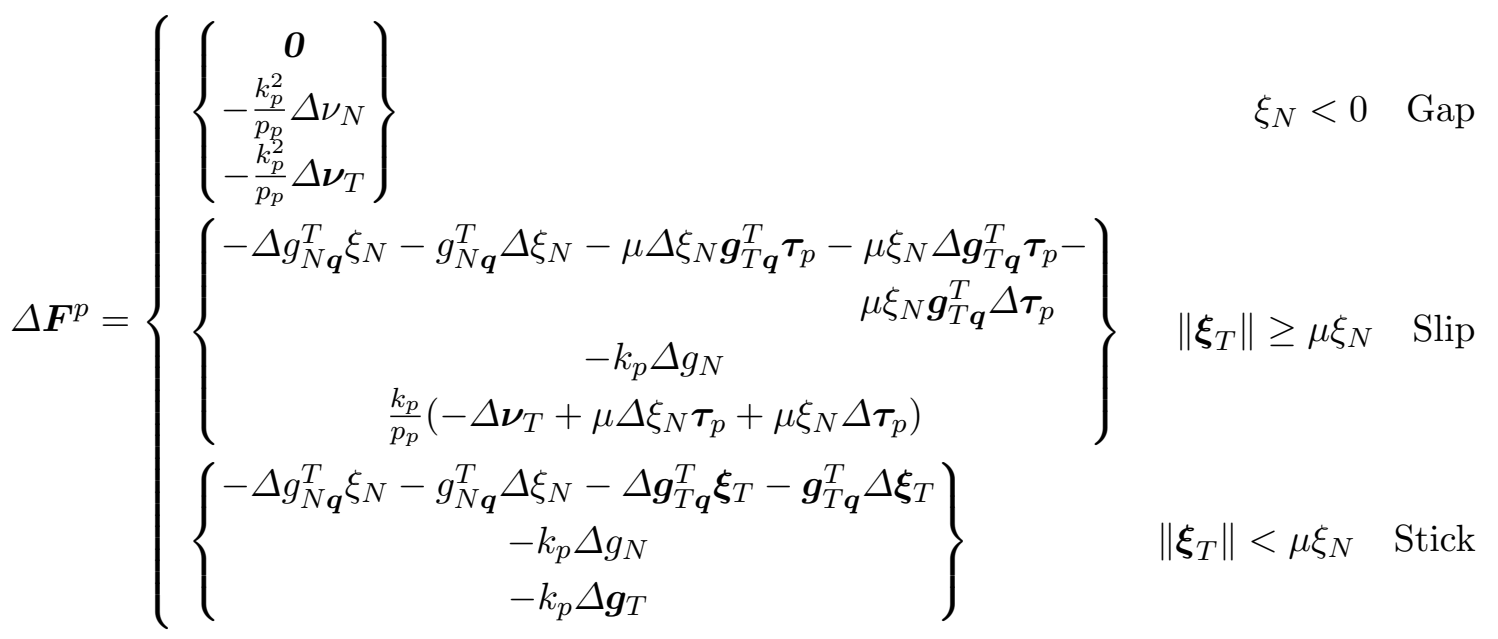

The contact/friction Hessian matrices are computed by replacing in Eq. (55) the Eqs. $(45,51)$ together with the derivatives of the matrices of constraints of gap and tangential displacements, which are next given:

$$
\begin{gathered}
\Delta g_{N \boldsymbol{q}}^{T}=\frac{\partial g_{N \boldsymbol{q}}^{T}}{\partial \boldsymbol{q}} \Delta \boldsymbol{q}=\left[\begin{array}{ccc}
\boldsymbol{0} & \boldsymbol{R}_{A} \widetilde{\boldsymbol{N}} & \boldsymbol{0} \\
-\widetilde{\boldsymbol{N}} \boldsymbol{R}_{A}^{T} & \widetilde{\boldsymbol{N}} \boldsymbol{d}_{A B} & \widetilde{\boldsymbol{N}} \boldsymbol{R}_{A}^{T} \\
\boldsymbol{0} & -\boldsymbol{R}_{A} \widetilde{\boldsymbol{N}} & \boldsymbol{0}
\end{array}\right]\left[\begin{array}{c}
\Delta \boldsymbol{x}_{A} \\
\Delta \boldsymbol{\Theta}_{A} \\
\Delta \boldsymbol{x}_{B}
\end{array}\right] \\
\Delta \boldsymbol{g}_{T \boldsymbol{q}}^{T} \boldsymbol{\tau}=\frac{\partial \boldsymbol{g}_{T \boldsymbol{q}}^{T}}{\partial \boldsymbol{\tau}} \Delta \boldsymbol{q}=\left[\begin{array}{ccc}
\boldsymbol{0} & \boldsymbol{R}_{A} \widetilde{\boldsymbol{\tau}} & \boldsymbol{0} \\
-\widetilde{\boldsymbol{\tau}} \boldsymbol{R}_{A}^{T} & \widetilde{\boldsymbol{\tau}} \overrightarrow{\boldsymbol{d}}_{A B} & \widetilde{\boldsymbol{\tau}} \boldsymbol{R}_{A}^{T} \\
\boldsymbol{0} & -\boldsymbol{R}_{A} \widetilde{\boldsymbol{\tau}} & \boldsymbol{0}
\end{array}\right]\left[\begin{array}{c}
\Delta \boldsymbol{x}_{A} \\
\Delta \boldsymbol{\Theta}_{A} \\
\Delta \boldsymbol{x}_{B}
\end{array}\right]
\end{gathered}
$$

The derivative of $\boldsymbol{\tau}_{p}$ is given by

$$
\Delta \boldsymbol{\tau}_{p}=\left(\boldsymbol{I}-\boldsymbol{\tau}_{p} \otimes \boldsymbol{\tau}_{p}\right) \frac{\boldsymbol{\xi}_{T}}{\left\|\boldsymbol{\xi}_{T}\right\|}
$$

and finally, the rotation increment $\Delta \boldsymbol{\Theta}_{A}$ is such that

$$
\Delta \boldsymbol{R}_{A}=\boldsymbol{R}_{A} \widetilde{\Delta \Theta_{A}}
$$

\subsection{Frictional contact problem at velocity level}

The augmented Lagrangian which regularizes the frictional contact problem at velocity level is given by

$$
\begin{aligned}
& \mathcal{L}^{v}\left(\boldsymbol{v}_{n+1}, \boldsymbol{\Lambda}_{n+1}\right)=-k_{v} \stackrel{\circ}{g}_{N, n+1} \Lambda_{N, n+1}+\frac{p_{v}}{2}\left(\stackrel{\circ}{g}_{N, n+1}\right)^{2}- \\
& \quad \frac{1}{2 p_{v}} \operatorname{dist}^{2}\left[k_{v} \Lambda_{N, n+1}-p_{v} \stackrel{\circ}{g}_{N, n+1}, \mathbb{R}^{+}\right]-k_{v} \stackrel{\circ}{\boldsymbol{g}}_{T, n+1} \cdot \boldsymbol{\Lambda}_{T, n+1}+ \\
& \quad \frac{p_{v}}{2}\left\|\stackrel{\circ}{\boldsymbol{g}}_{T, n+1}\right\|^{2}-\frac{1}{2 p_{v}} \operatorname{dist}^{2}\left[k_{v} \boldsymbol{\Lambda}_{T, n+1}-p_{v} \stackrel{\circ}{\boldsymbol{g}}_{T, n+1}, C_{\sigma_{N}}\right]
\end{aligned}
$$


where $C_{\sigma_{N}}$ is a section of radius $\mu \sigma_{N}$ of the augmented Coulomb friction cone expressed in terms of variables at velocity level with the generalized velocity vector given by

$$
\boldsymbol{v}_{n+1}=\left[\dot{\boldsymbol{x}}_{A, n+1}^{T} \boldsymbol{\Omega}_{A, n+1}^{T} \dot{\boldsymbol{x}}_{B, n+1}^{T}\right]^{T}
$$

Additionally, the dependence of the impact law in terms of $\boldsymbol{v}$ is not explicitly specified in Eq. (60) for the sake of conciseness.

Then, by following a similar reasoning as that presented in Section 4.1, the internal force vectors at velocity level are given by

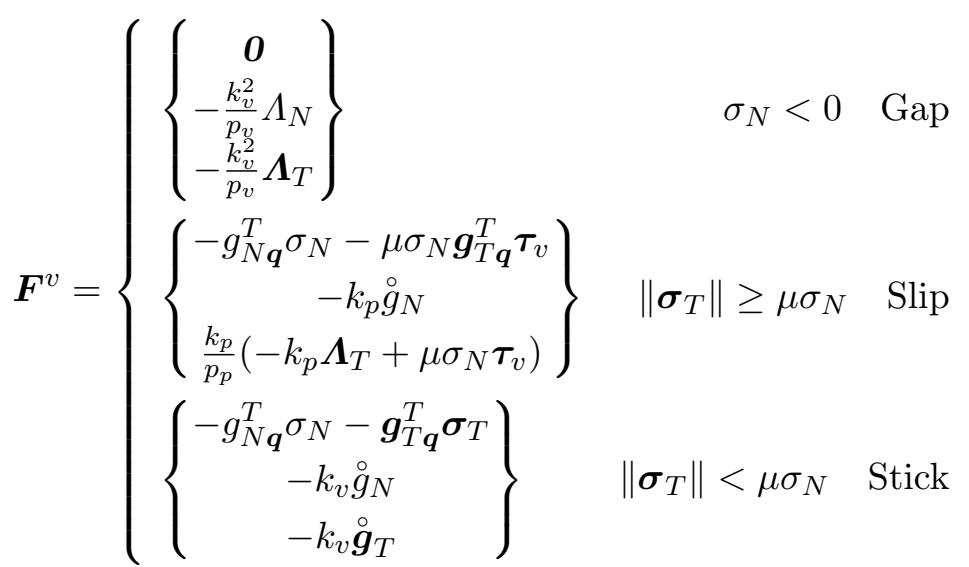

The unit vector $\boldsymbol{\tau}_{v}$ pointing in the direction of the contact force is defined as $\boldsymbol{\tau}_{v}=\boldsymbol{\Lambda}_{T} /\left\|\boldsymbol{\Lambda}_{T}\right\|$.

Finally, the linearization of the internal force vector gives the Hessian matrix. Note that in Step 3 of the algorithm presented in Section 2, where the projection on velocity constraints are calculated, the variable $\boldsymbol{q}$ coming from the solution of the position sub-problem is fixed. Thus, the gradient matrices $g_{N \boldsymbol{q}}$ and $\boldsymbol{g}_{T \boldsymbol{q}}$ are constant at this instance and do not contribute in the calculation of the Hessian matrix. Therefore, the Hessian matrix for the different contact conditions is as follows:

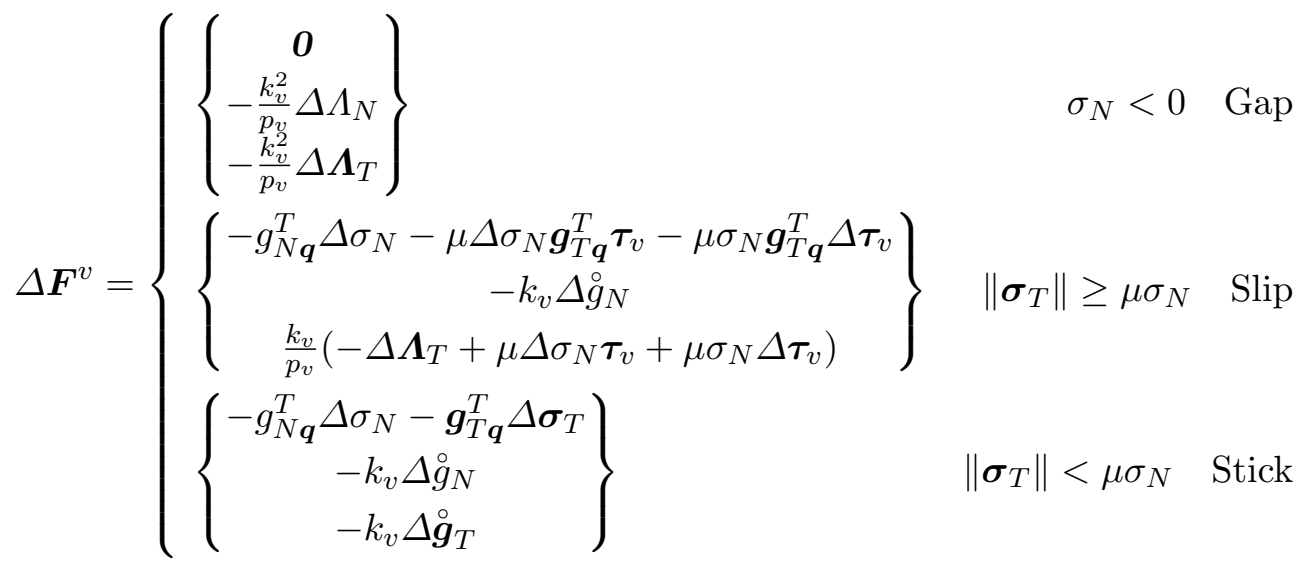


The contact/friction Hessian matrices are computed similarly to the contact/friction Hessian matrices at the position level. The internal forces vectors and the Hessian matrices at the position and velocity levels contribute to the global tangent matrices and to the generalized internal forces vectors by a standard assembly procedure.

\section{$5 \quad$ Numerical examples}

Several numerical examples are studied to evaluate the robustness and accuracy of the proposed frictional contact model. Each of the examples has been chosen to highlight different characteristics that help to evaluate the performance of the proposed friction algorithm. In the first example, a point mass undergoes slipping and sticking motion on an inclined plane, showing the ability of the proposed method to handle a friction force different from zero at equilibrium state. The second example consists of a rocking rod impacting two supports, which allows us to assess the performance of the algorithm when the contact surface does not remain still. Then, in order to study problems with flexible components, the motion of a sliding chain of springs and masses and the oblique impact of a beam with a rigid wall are considered. The last example consists in the simulation of a pendulum impacting a rigid plane attached to flexible supports, showing the ability of the algorithm for handling flexible elements, a moving contacting surface and 3D trajectories which imply a 3D frictional behaviour.

The frictional contact algorithm proposed in this work has been implemented in the finite element code Oofelie [29]. The convergence of the numerical solution obtained with the proposed methodology is analyzed in all examples. For that purpose, the error for each value of the time increment $h$ is evaluated using the $L^{1}$ norm:

$$
\operatorname{Error}(h)=\frac{\sum_{i=0}^{N}\left|f_{i}-f\left(t_{i}\right)\right|}{\sum_{i=0}^{N}\left|f\left(t_{i}\right)\right|}
$$

where $N$ is the number of time steps, $f_{i}$ is the numerical solution obtained using the nonsmooth generalized- $\alpha$ method (NSGA) and $f\left(t_{i}\right)$ is the reference solution. The analytical solution is adopted as a reference solution in all cases in which it is available, otherwise the numerical solution corresponding to a very small time increment is taken as reference solution.

\subsection{A point mass sliding and sticking on an inclined plane}

This example intends to show the ability of the proposed algorithm to handle the switch between the sliding and sticking states, and to handle cases with a friction force different from zero at equilibrium state. The problem consists of a point mass which is initially in sliding state with an initial tangential 
velocity of $v_{0}=2 \mathrm{~m} / \mathrm{s}$ with respect to the inclined plane, Fig. 3(a). Under the action of gravity and friction, the mass looses velocity. The final state depends on the friction coefficient and on the plane angle values. Here, the friction coefficient is larger than the tangent of the slope angle. Therefore, the mass reaches a state of sticking.

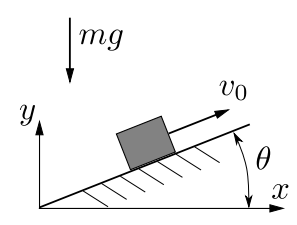

(a)

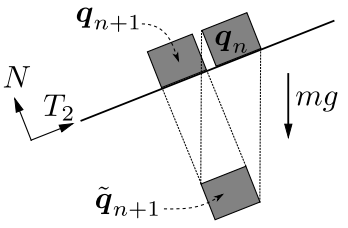

(b)

Figure 3: (a) A point mass sliding and sticking on an inclined plane. (b) Position correction when Coulomb's friction law is not imposed at position level.

The parameters used for the simulation are defined as follows: the mass of the point is $m=1 \mathrm{~kg}$, the angle of the inclined plane is $\pi / 12$, the gravity acceleration is $g=9.81 \mathrm{~m} / \mathrm{s}^{2}$, the friction coefficient is $\mu=0.3$ and the restitution coefficients for the normal and tangential directions are zero. The numerical solution is computed with a spectral radius of $\rho_{\infty}=0.8$ and a total simulation time of $2 \mathrm{~s}$.

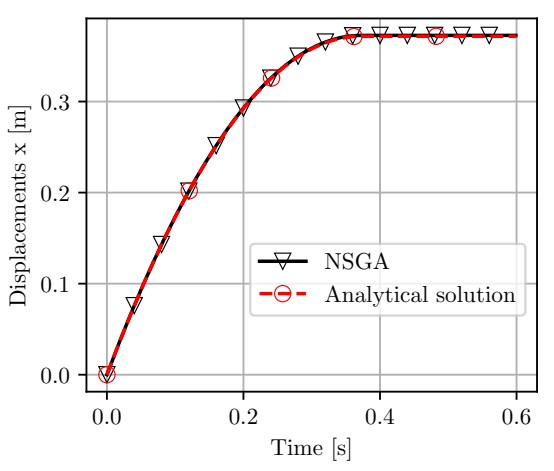

(a) Displacement in $x$ direction.

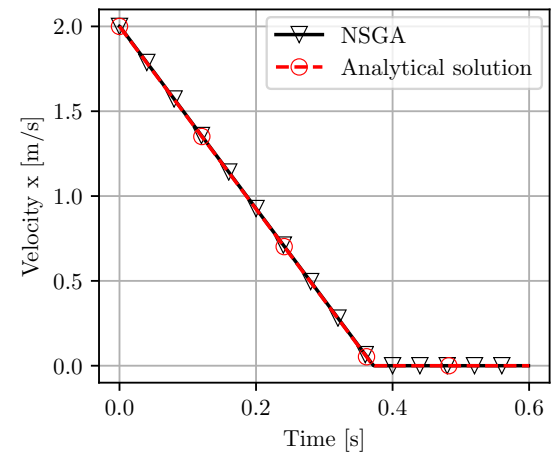

(b) Velocity in $x$ direction.

Figure 4: Point mass sliding and sticking on an inclined plane: results for a time step of $10^{-3} \mathrm{~s}$. 


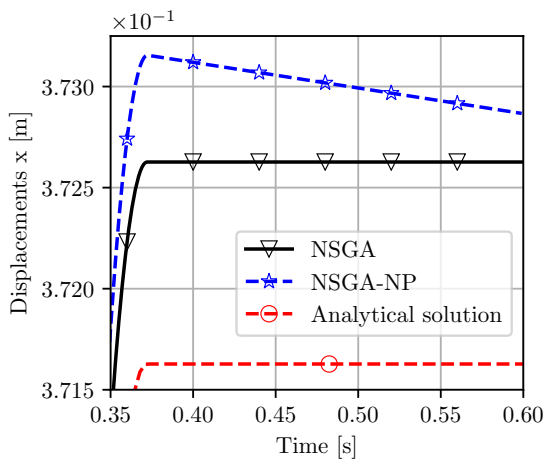

(a) Displacement in $x$ direction.

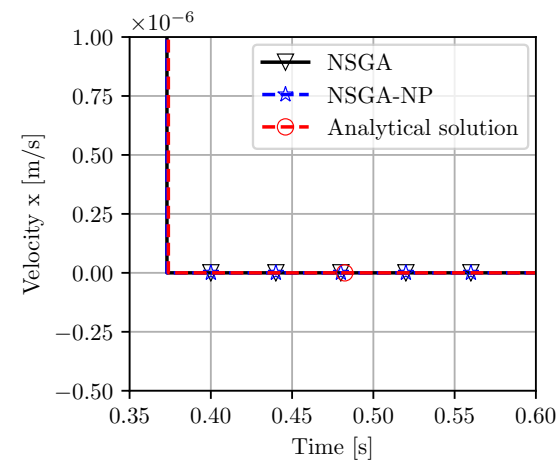

(b) Velocity in $x$ direction.

Figure 5: Zoom of the Fig.4, adding also the results for the case of NSGA-NP

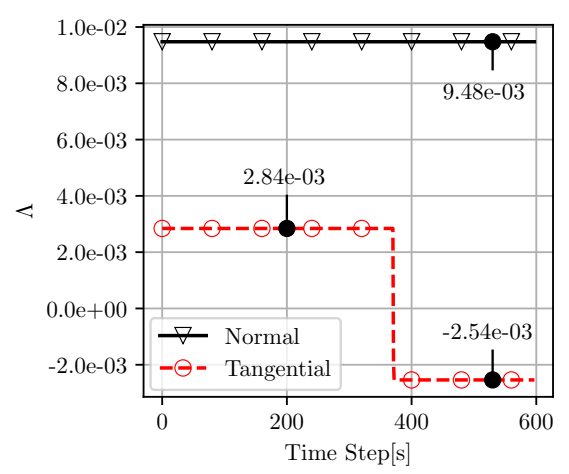

(a) Lagrange multipliers.

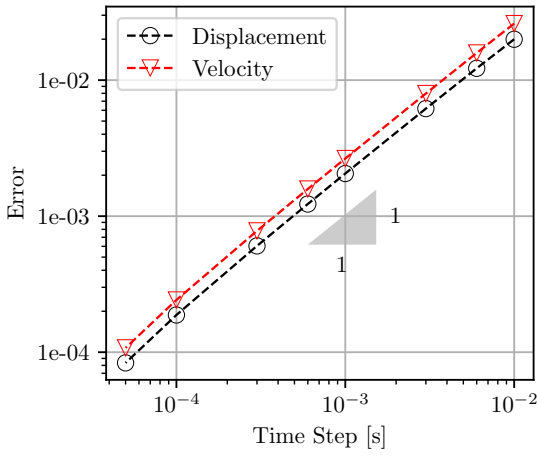

(b) Convergence rate curves.

Figure 6: Point mass sliding and sticking on an inclined plane: results for a time step of $10^{-3} \mathrm{~s}$.

A comparison between the numerical and analytical solutions for the displacement and the velocity fields is shown in Figs. 4(a,b), respectively, where a good agreement between them can be noticed. As expected, it can be observed that a slip motion takes place from $0 \mathrm{~s}$ to $0.374 \mathrm{~s}$, followed by a constant sticking phase in which the block remains at rest.

As already mentioned in Section 4.1, the Coulomb's friction law must also be imposed at position level, due to the fact that both $\tilde{\boldsymbol{q}}$ and $\boldsymbol{U}$ are blind to any correction done at velocity level during the time step. In Fig. 5(a), the results obtained for the $x$ component of the position and the velocity for the case in which the Coulomb's friction law is not imposed at position level (NSGA-NP) are shown. As it can be observed in the NSGA-NP solution, when the contact is in stick mode, the position still evolves even though the velocity is zero. This non-physical phenomenon of the NSGA-NP algorithm 
is explained in Fig. 3(b), where it is made clear that the smooth prediction of the position $\tilde{\boldsymbol{q}}$ is only corrected in the normal direction, and, therefore, the position of the block ends up artificially sliding down in a kind of numerical drift. This problem is reduced when the time step of the NSGA-NP algorithm decreases and fully disappears when imposing in the NSGA algorithm as the Coulomb's law is then imposed at position level, see Figs. 4 and 5 .

In Fig. 6(a) the Lagrange multipliers $\boldsymbol{\Lambda}$ in the normal and tangential directions can be observed. As expected from the physics, in the tangential direction a sign change takes place and it represents the change of the contact status, from sliding to sticking. Additionally, once the mass is at rest the numerical solution agrees with the analytical values for the normal reaction impulse $\Lambda_{N}$ and the tangential friction impulse $\Lambda_{T}$ which are in this case $9.48 \times 10^{-3} \mathrm{Ns}$ and $-2.54 \times 10^{-3} \mathrm{Ns}$, respectively. During the sliding phase the value of the tangential impulse is $\Lambda_{T}=2.84 \times 10^{-3} \mathrm{Ns}$, which is equal to $\mu \Lambda_{N}$, satisfying the Coulomb's frictional law. It is remarked that in order to obtain the constraint force, the Lagrange multiplier $\boldsymbol{\Lambda}$ must be divided by the time step $h$, because $\boldsymbol{\Lambda}$ represents the impulse of the unilateral constraints which is the integral of the reaction force in the time interval $\left(t_{n}, t_{n+1}\right]$. Lastly, Fig. 6(b) shows the convergence obtained for the displacement and velocity fields, where, as expected, $\mathcal{O}(h)$ is achieved.

\section{$5.2 \quad$ A rocking rod impacting two supports}

Let us study a rigid rocking rod impacting two supports, see Fig. 7, which has already been studied by several authors [52-54]. In this example, the contact occurs between the support points and the face represented by the rod, meaning that the contact surface does not remain still. The parameters of the model and the initial conditions are taken from Zander et al. [52]. The rocking rod initially falls from a height $h_{1}=0.104 \mathrm{~m}$ with an initial angle $\theta=12^{\circ}$ under the action of gravity $g=9.8 \mathrm{~m} / \mathrm{s}^{2}$. Then, it impacts against two rigid obstacles separated by a horizontal distance $a$ of $0.4 \mathrm{~m}$ and a vertical height $h_{2}$, as depicted in Fig. 7. The rod is considered rigid and slender; it has a length $l=1 \mathrm{~m}$, an inertia moment $I_{z}=4 \times 10^{-2} \mathrm{~kg} \mathrm{~m}^{2}$ and a mass $m=0.48 \mathrm{~kg}$. Zander et al. [52] made a numerical study to find the restitution coefficients that best approximate the results obtained using a flexible model of the rod, and they found the values for the normal and tangential restitution coefficients to be $e_{N}=0.6262$ and $e_{T}=0$, respectively. In the following simulations, the time increment is $10^{-3} \mathrm{~s}$, the spectral radius is $\rho_{\infty}=0.8$ and the total simulation time is $0.3 \mathrm{~s}$. 


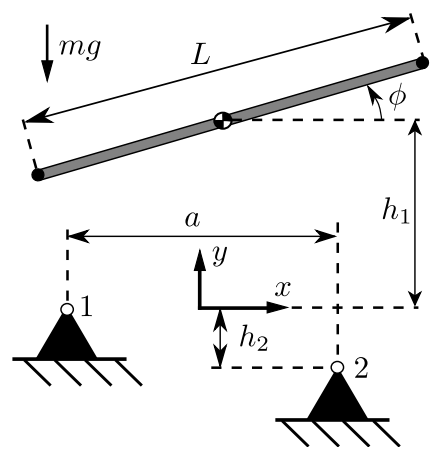

Figure 7: Rocking rod dimensions.

In order to evaluate the robustness of the proposed methodology, four different configurations for the supports are considered. The vertical position of the supports and the friction coefficients are selected as specified in Table 1 , where $h_{2}$ is the vertical distance between supports and, $\mu_{1}$ and $\mu_{2}$ are the friction coefficients at support 1 and 2, respectively.

\begin{tabular}{ccccc}
\hline Case & $h_{2}[\mathrm{~m}]$ & $\mu_{1}$ & $\mu_{2}$ & $a[\mathrm{~m}]$ \\
\hline I & 0 & 0.1 & 0.1 & 0.4 \\
II & 0 & 0 & 0.1 & 0.4 \\
III & 0.4 & 0.3 & 0.3 & 0.4 \\
IV & 0.4 & 0 & 0.3 & 0.4 \\
\hline
\end{tabular}

Table 1: Rocking rod parameters. 


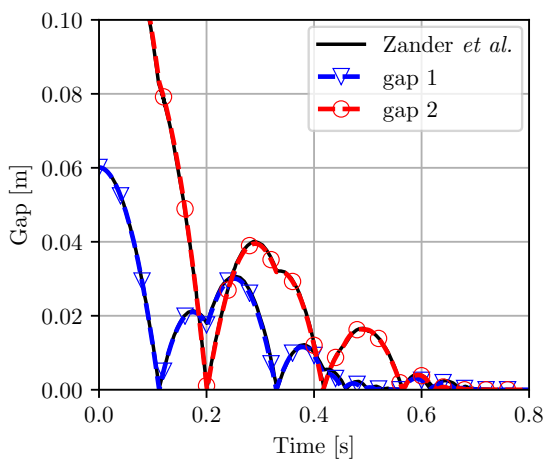

(a) Case I.

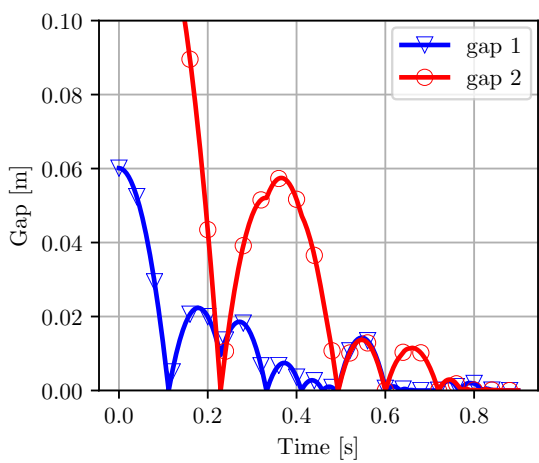

(c) Case III.

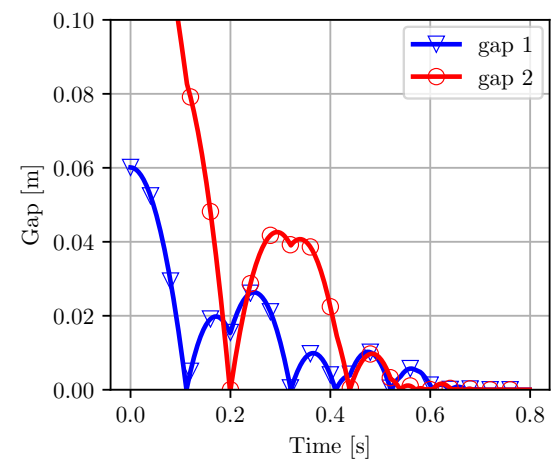

(b) Case II.

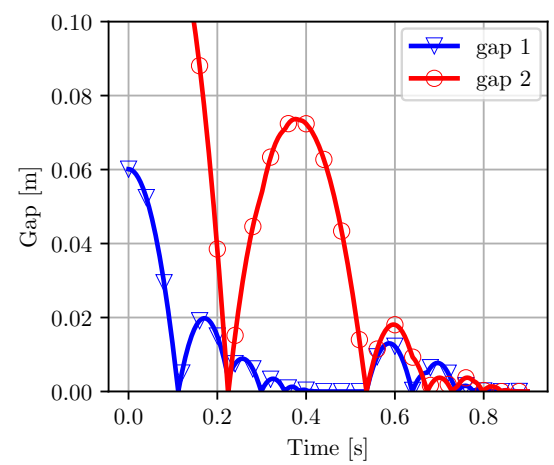

(d) Case IV.

Figure 8: Normal gap in the contacts for the rocking rod example.

The numerical solution of Case I is depicted in Fig. 8(a), where the normal gap distance between the supports and the rod are compared to the solution computed by Zander et al. [52]. Both solutions are in a good agreement. Figure 8(b) presents the results for Case II, where the normal gap distance at support 1 is assumed frictionless, whilst friction effects are considered in the support 2. By analyzing this figure, after the first impact, the normal gap distance at support 1 shows different bounces from the frictionless case (take Fig. 8(a) as reference). On the other hand, the solution for the normal gap distance at support 2 is not very affected until impacting for the second time. This happens because during this period of time the right end of the rod is in a free fall motion. Then, after the third impact, the solutions for Case I and II show a different behaviour.

The cases II and III, in which the supports are not aligned horizontally and the friction coefficients are different, are also tested. It can be observed that the solutions in Figs. 8(c,d) are quite different compared with Cases I and II. In all the studied cases, exact satisfaction of the constraints at 


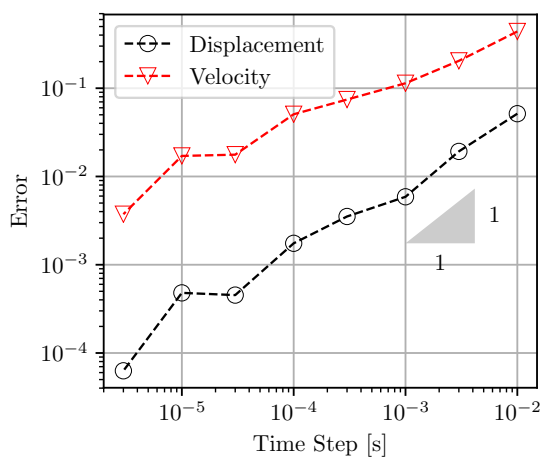

(a) Case I.

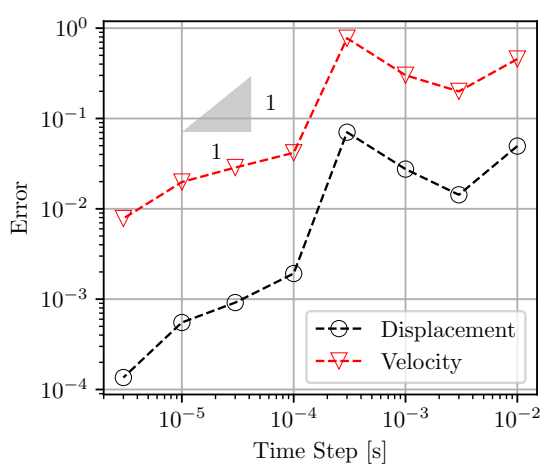

(c) Case III.

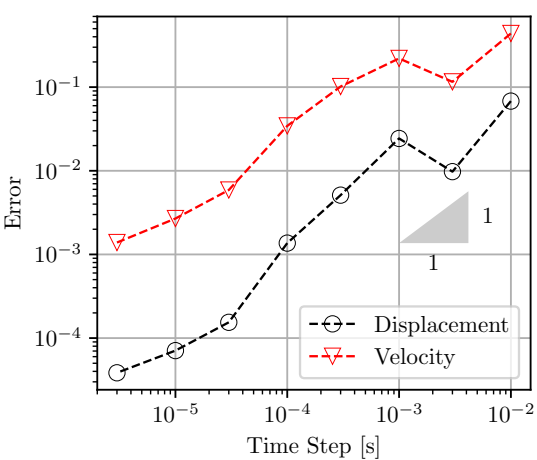

(b) Case II.

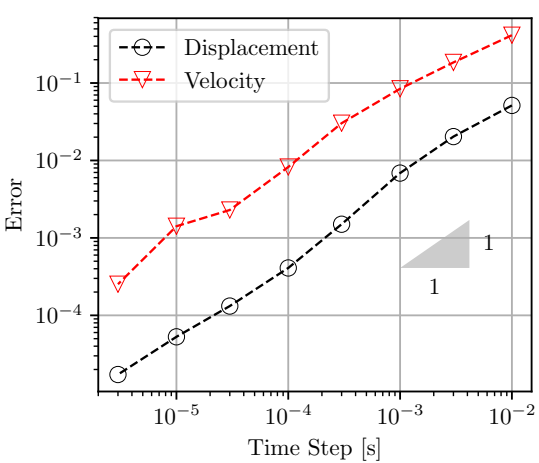

(d) Case IV.

Figure 9: Convergence rate for the rocking rod example.

position level is attained, see Fig. 8.

The convergence of the error with the time step is shown in Fig. 9, the reference solution is obtained with $h=10^{-6} \mathrm{~s}$. A linear convergence rate is obtained for all cases.

\subsection{Sliding masses connected by springs}

In this example, the capability of the proposed methodology to deal with flexible components is studied. It consists of 21 identical masses, $m=$ $0.0025 \mathrm{~kg}$, connected by 20 equal springs of length $\ell=0.05 \mathrm{~m}$ and rigidity $k=20 \mathrm{~N} / \mathrm{m}$, as shown in Fig. 10. The system has zero initial velocity and displacement, with an axial force $F=0.4 \mathrm{~N}$ acting on the first node from $t=$ $0 \mathrm{~s}$ to $t=0.1 \mathrm{~s}$. A friction coefficient $\mu=0.3$ and a gravity acceleration of $g=$ $9.81 \mathrm{~m} / \mathrm{s}^{2}$ are adopted. The normal and tangential restitution coefficients are zero, and the analyzed time interval is $t \in[0,1.5] \mathrm{s}$. 


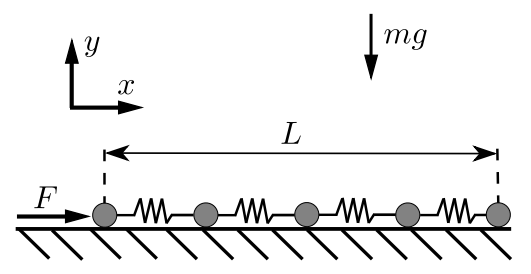

Figure 10: System of sliding masses connected by springs.

First, a time step of $h=10^{-3} \mathrm{~s}$ is used. Fig. 11(a) shows the positions of the first and last nodes, and the total length of the system. The first node starts to move in response to the action of the applied force and a wave front propagates. The last node starts to move after $0.22 \mathrm{~s}$, with the subsequent shrinkage of the system and a wave reflection. Thanks to friction, the wave propagates in several cycles but with a decreasing amplitude up to the point where the system remains still without moving and with its initial length recovered. The wave propagation effect can be clearly observed in Fig. 11(b) as well as in Fig. 12(a) where the velocity for each node is shown as a function of time. Finally, the results for a convergence study can be observed in Fig. 12(b) where, as expected, $\mathcal{O}(h)$ is achieved.

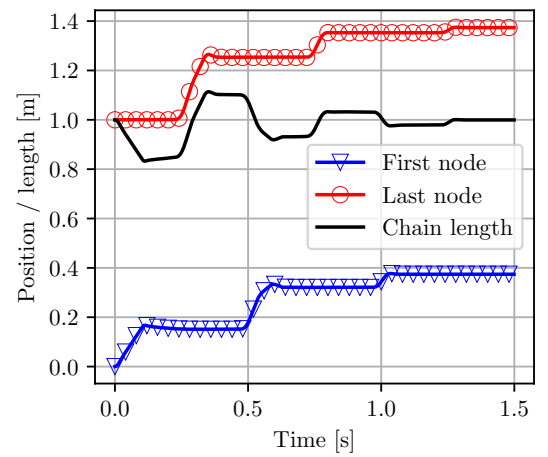

(a) Nodes position.

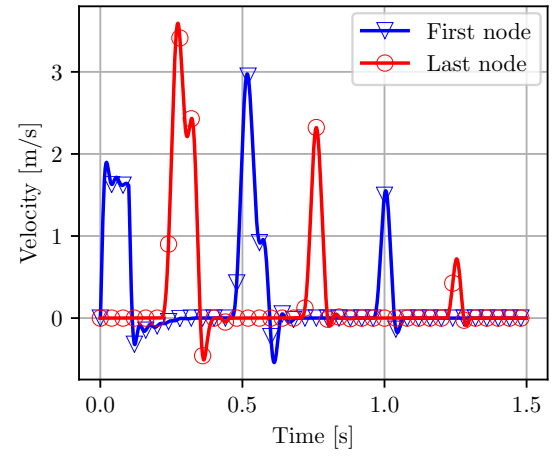

(b) Nodes velocity.

Figure 11: Sliding masses for a time step $h=10^{-3}$ s. 


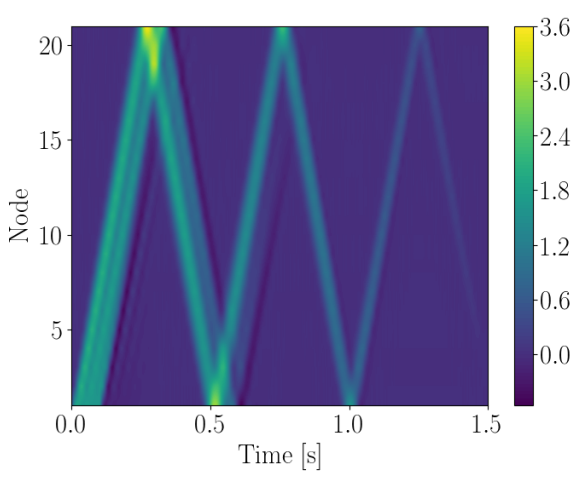

(a) Plot of nodal velocity amplitudes in time, illustrating wave propagation $(h=$ $\left.10^{-3} \mathrm{~s}\right)$.

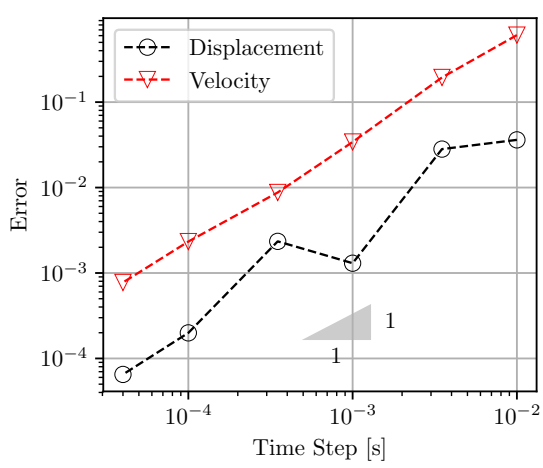

(b) Convergence study.

Figure 12: Sliding masses results.

\subsection{Oblique impact of an elastic beam against a rigid wall}

This example deals with the oblique impact of an elastic beam against a rigid wall (Fig. 13), which allows us to evaluate the robustness of the method for problems involving friction and nonlinear flexible elements such as beams. Initially, García Orden and Goicolea [55] studied this problem using truss elements and only axial deformation effects. Then, Lens and Cardona [56] proposed a modification taking into account the flexural behaviour by using beam elements in the framework of an energy preserving time integration scheme.

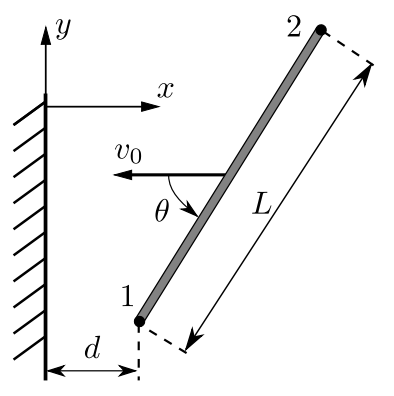

Figure 13: Oblique impact of a flexible beam with a rigid wall.

The elastic beam impacts against a vertical rigid wall with an angle of incidence $\theta$. It moves horizontally until the lower tip of the beam impacts with the rigid wall. Then, it rotates resulting in a second impact at the upper end of the beam. The initial configuration is defined by $\theta=35.2^{\circ}$ and 
$v_{0}=2 \mathrm{~m} / \mathrm{s}$. The beam is $1 \mathrm{~m}$ long with a transverse area of $2.5477 \times 10^{-3} \mathrm{~m}^{2}$ and inertia moment of $I=5.40897 \times 10^{-7} \mathrm{~m}^{4}$. The Young modulus is $1 \times$ $10^{9} \mathrm{~N} / \mathrm{m}^{2}$, the Poisson ratio is 0.3 and the mass density is $\rho=7850 \mathrm{~kg} / \mathrm{m}^{3}$. The friction coefficient is $\mu=0.5$ and the normal and tangential restitution coefficients are zero. The beam is discretized with eight equally spaced nonlinear beam finite elements.

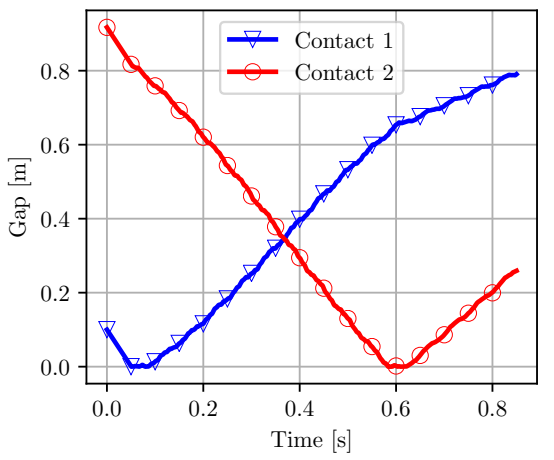

(a) Normal gap.

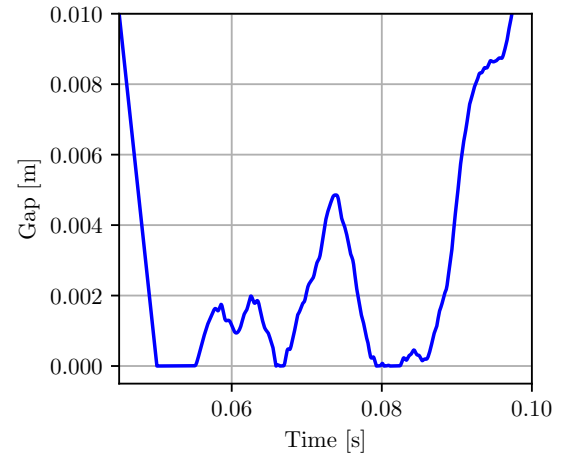

(b) Zoom of the normal gap for contact 1 .

Figure 14: Oblique impact of a beam results.

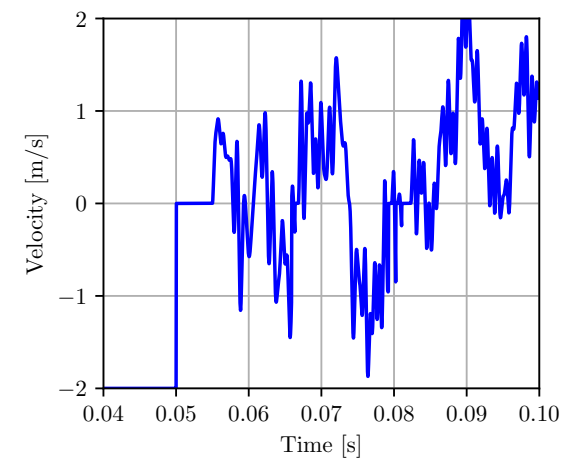

(a) Horizontal velocity of the lower end of the beam.

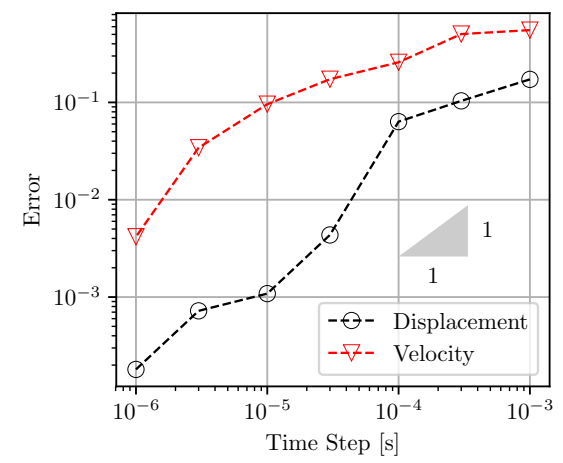

(b) Convergence study.

Figure 15: Oblique impact of a beam results.

The normal gap for both contacts are shown in Fig. 14(a), where the results are computed with a time step $h=10^{-4}$. It can be observed that the first impact takes place in the lower end of the beam at $0.05 \mathrm{~s}$. The second impact occurs at the other end of the beam at $0.5878 \mathrm{~s}$. A detail of the motion involving the dynamics of the first impact is shown in 14(b) and 15(a). It is observed that the impact phase has a duration of $82.5 \mathrm{~ms}$. 
During the first $5 \mathrm{~ms}$ the contact remains closed, after which the tip of the beam clatters until the contact is completely released. This vibration effect is more evident at the velocity level, as it can be appreciated in Fig. $15(\mathrm{a})$, where the $x$-component of the velocity field during the impact phase is shown. The convergence analysis is shown in Fig. 15(b), it was computed for a total time of $0.85 \mathrm{~s}$ with different time step sizes.

The numerical solutions presented in this paper can be compared to those presented by Lens and Cardona [56] or García Orden and Goicolea [55]. However, we should take into account that García Orden and Goicolea only considered axial deformation effects and friction was not modelled. On the other hand, in the model of Lens and Cardona [56] the flexural behaviour was modeled using beam elements; however, the impact was frictionless. Furthermore, in both cases, the impact equations were regularized by the penalty method, and a high dependency of results on the penalty factor was shown. Hence, owing to these facts, the dynamic response obtained with the current model is not exactly the same to the ones found in $[55,56]$.

\subsection{A pendulum impacting a rigid plane attached to flexible supports}

A pendulum impacting a rigid plane attached to flexible supports as shown in Fig. 16 has been simulated. The plane is of square shape with a side length of $l=5 \mathrm{~m}$. Its center of mass, located at its geometrical center, has its translations fixed, therefore it can only rotate. Each of the four corners of this plane are attached to a geometrically nonlinear spring-damper element, where the stiffness constant and the damping coefficient are $100 \mathrm{~N} / \mathrm{m}$ and $1 \mathrm{Ns} / \mathrm{m}$, respectively, and the initial length is $L_{0}=0.2 \mathrm{~m}$. The mass and the principal moments of inertia of the plate are equal to $m_{s}=0.2 \mathrm{~kg}$ and $I_{s}=m_{s} L^{2} / 6$. At a height $h_{0}=1 \mathrm{~m}$ from the center of the plane, there is attached a pendulum of $1.3 \mathrm{~m}$ length with mass and principal moments of inertia equal to $m_{p}=5 \mathrm{~kg}$ and $I_{p}=m_{p} l^{2}$. The fixed end of the pendulum is free to rotate, and initially the free end is in the position $(0 ; 1.3 ; 1)$ $\mathrm{m}$. Both, the pendulum and the plane are under the action of the gravity equal to $9.81 \mathrm{~m} / \mathrm{s}^{2}$. Initially, the pendulum has an angular velocity of $\Omega=(0 ; 0 ; 1.5) \mathrm{rad} / \mathrm{s}$ and the free end a velocity of $\boldsymbol{v}=(-1.95 ; 0 ; 0) \mathrm{m} / \mathrm{s}$. Friction is modelled adopting a friction coefficient equal to $\mu=0.3$, and the normal and tangential restitution coefficients are taken equal to zero. This setting allows us to show the ability of the proposed algorithm for handling flexible elements and a 3D frictional behaviour. 


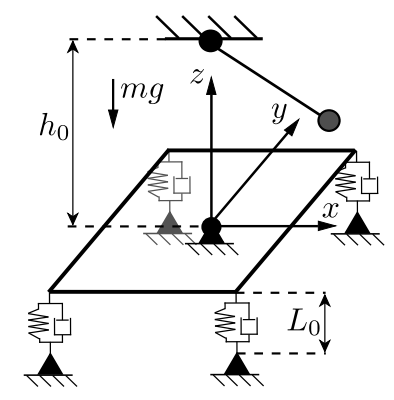

Figure 16: Pendulum impacting a rigid plane attached to flexible supports: Configuration of the problem.

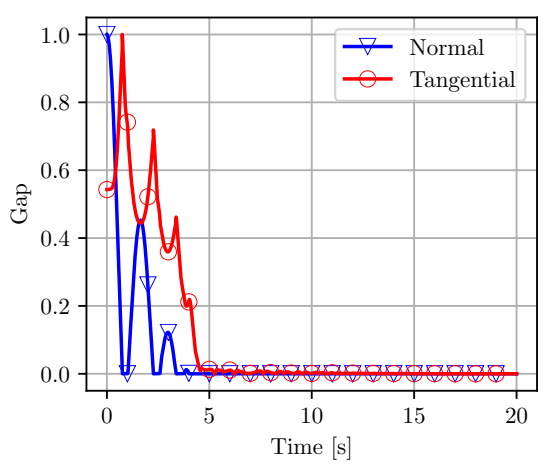

(a) Gap in the normal direction and $L^{2}$ norm of the Gap in the tangential directions.

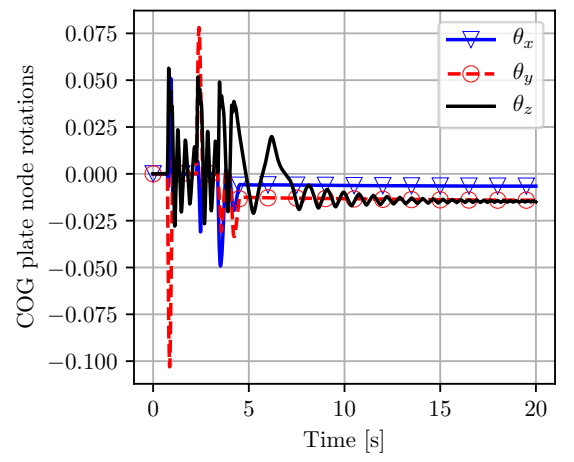

(c) Rotation vector of the plane.

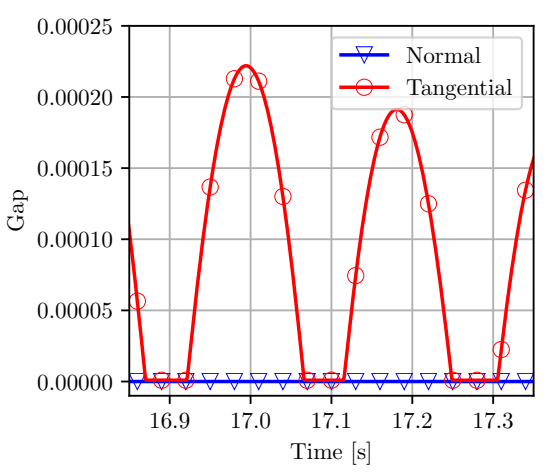

(b) Zoom of the previous figure.

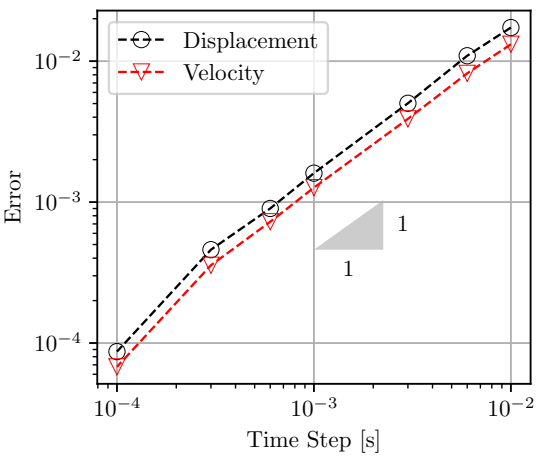

(d) Convergence study.

Figure 17: Pendulum impacting a rigid plane attached to flexible supports (results for $h=10^{-3}$ ).

The pendulum starts a downwards trajectory in response to the effect 
of gravity, until colliding with the plane attached to flexible supports. Due to the flexibility of the system, the pendulum bounces adopting states of sliding and sticking as the orientation of the plane changes in response to the collisions with the pendulum. This can be observed in Fig. 17(a-b), where the bounces of the pendulum can be reckoned by analyzing the normal component of the gap, and the sticking and sliding phases can be inferred from the graph of the norm of the gap in the tangential direction. In Fig. 17 (c) the history of the rotation vector of the plane can be appreciated. The results for a convergence study are shown in Fig. 17(d) where, as expected, $\mathcal{O}(h)$ is achieved.

\section{Conclusions}

In this work, a new frictional contact algorithm for nonsmooth multibody systems is presented. The integration of the equations of motion of the frictional contact problem is performed using the nonsmooth generalized- $\alpha$ time integration scheme based on an augmented Lagrangian approach. The smooth contributions are integrated using a second-order scheme, whereas a first order scheme is used for the impulsive contributions. Compared with the classical Moreau-Jean method, the proposed method leads to qualitatively better numerical solutions with less numerical dissipation. The proposed contact model satisfies exactly the contact constraints at position level, which means that no penetration is observed. Impacts are also properly solved with the exact satisfaction of constraints at velocity level. Then, the complementarity problem for the normal and frictional contact problem is solved at each time step using a Newton semi-smooth method in a fully implicit approach with fast convergence. The proposed methodology has been successfully applied to study different mechanism configurations consisting of flexible and/or rigid bodies and to observe the friction effects on the dynamic response of the system.

The presented numerical examples demonstrated the ability of the scheme to represent frictional contact problems with large displacements and rotations in a two and three dimensions. The algorithm has three main features: i) the final solution is independent of the penalty parameters both in the tangential and normal directions, their value mostly affect the convergence rate, ii) the algorithm does not require modifications in existing finite elements of the library, in other words it is minimally intrusive and iii) the smooth motion of a mechanism is solved using a generalized $-\alpha$ method that allows to integrate the equations of the flexible components with second-order accuracy and with controlled dissipation, something that is not possible with the first order integrators which are usually used to solve this kind of problems and introduce a much higher numerical dissipation. The equations for the analytical computation of the residual forces and tangent matrices of 
the frictional contact algorithm are provided. Five numerical examples of mechanisms with unilateral friction constraints are presented. Finally, the results of these examples were compared to analytical equations and previous numerical solutions obtained by other authors showing good agreement and convergence rate.

\section{Acknowledgements}

This work received financial support from Consejo Nacional de Investigaciones Científicas y Técnicas (CONICET), Universidad Nacional del Litoral, Agencia Nacional de Promoción Científica y Tecnológica (ANPCyT) PICT20151067, Universidad Tecnológica Nacional PID-UTN UTI4790TC, and the M4 project funded by the Walloon Region (Pôle MecaTech), which are gratefully acknowledged.

\section{References}

[1] Duriez, C., Dubois, F., Kheddar, A., and Andriot, C., 2006. "Realistic haptic rendering of interacting deformable objects in virtual environments". IEEE Transactions on Visualization and Computer Graphics, 12, pp. 0-47.

[2] Pfeiffer, F., and Glocker, C., 1996. Multibody dynamics with unilateral contacts. Wiley series in nonlinear science. Wiley.

[3] Acary, V., 2013. "Projected event-capturing time-stepping schemes for nonsmooth mechanical systems with unilateral contact and coulomb friction". Computer Methods in Applied Mechanics and Engineering, 256, pp. $224-250$.

[4] Johnson, K., 1987. Contact Mechanics. Cambridge University Press.

[5] Pfeiffer, F., 2008. "On non-smooth dynamics". Meccanica, 43, pp. 533554.

[6] Esche, S., Kinzel, G., and Altan, T., 1997. "Issues in convergence improvement for non-linear finite element programs". International Journal for Numerical Methods in Engineering, 40, pp. 4577-4594.

[7] Bostan, M., and Hild, P., 2009. "Weak formulations and solution multiplicity of equilibrium configurations with Coulomb friction". Mathematical Modelling of Natural Phenomena, 4, pp. 147-162.

[8] Pennestrí, E., Rossi, V., Salvini, P., and Valentini, P. P., 2016. "Review and comparison of dry friction force models". Nonlinear Dyn, 83, pp. $1785-1801$. 
[9] Marques, F., Flores, P., Claro, J. C., and Lankarani, H. M., 2019. "Modeling and analysis of friction including rolling effects in multibody dynamics: a review". Multibody System Dynamics, 45, pp. 223-244.

[10] Acary, V., Brémond, M., and Huber, O., 2018. On Solving Contact Problems with Coulomb Friction: Formulations and Numerical Comparisons. Springer International Publishing, Cham, pp. 375-457.

[11] Kikuchi, N., and Oden, J., 1988. Contact Problems in Elasticity: A Study of variational Inequalities Constrains and Finite Element Method. SIAM, Philadelphia.

[12] Belytschko, T., and Neal, M., 1991. "Contact-impact by the pinball algorithm with penalty and Lagrangian-methods". International Journal for Numerical Methods in Engineering, 31, pp. 547-572.

[13] Wriggers, P., 2002. Computational Contact Mechanics. John Wiley and Sons.

[14] Cavalieri, F., Fachinotti, V., and Cardona, A., 2012. "A mortar contact algorithm for three-dimensional elasticity problems". Revista Internacional de Métodos Numéricos para Cálculo y Diseño en Ingeniera, 28(2), pp. 80-92.

[15] Lankarani, H., and Nikravesh, P., 1994. "Continuous contact force models for impact analysis in multibody analysis". Nonlinear Dynamics, 5, pp. 193-207.

[16] Brogliato, B., 1999. Nonsmooth Mechanics. Springer.

[17] Acary, V., 2012. "Higher order event capturing time-stepping schemes for nonsmooth multibody systems with unilateral constraints and impacts". Applied Numerical Mathematics, 62(10), pp. 1259 - 1275.

[18] Simo, J., and Laursen, T., 1992. "An augmented Lagrangian treatment of contact problems involving friction". Computers and Structures, 42(1), pp. $97-116$.

[19] Love, G., and Laursen, T., 2003. "Improved implicit integrators for transient impact problems-dynamic frictional dissipation within an admissible conserving framework". Computer Methods in Applied Mechanics and Engineering, 192(19), pp. 2223 - 2248.

[20] Areias, P., César de Sá, J., and Conceição António, C. A., 2004. "Algorithms for the analysis of 3D finite strain contact problems". International Journal for Numerical Methods in Engineering, 61, pp. 11071151. 
[21] Alart, P., and Curnier, A., 1991. "A mixed formulation for frictional contact problems prone to Newton like solution methods". Computer Methods in Applied Mechanics and Engineering, 92(3), pp. 353-375.

[22] Hestenes, M., 1969. "Multiplier and gradient methods". J. Optim. Theory and Applic., 4, pp. 303-320.

[23] Powell, M., 1969. "A method for nonlinear constraints in optimization problems. in: Fletcher R. (ed) optimization". Academic Press, London., pp. $283-298$.

[24] Géradin, M., and Cardona, A., 2001. Flexible Multibody Dynamics: A Finite Element Approach. Wiley.

[25] Moreau, J., 1988. Unilateral Contact and Dry Friction in Finite Freedom Dynamics. Springer Vienna, Vienna, pp. 1-82.

[26] Jean, M., 1999. "The non-smooth contact dynamics method". Computer Methods in Applied Mechanics and Engineering, 177(3), pp. 235257.

[27] Dubois, F., Jean, M., Renuf, M., Mozul, R., Martin, A., and Bagneris, M., 2011. LMGC90. CSMA.

[28] Acary, V., and Pérignon, F., 2007. An introduction to SICONS. INRIA. Tech. Rep. 0340.

[29] Cardona, A., Klapka, I., and Géradin, M., 1994. "Design of a new finite element programming environment". Engineering Computations, 11, pp. 365-381.

[30] Newmark, N., 1959. "A method for computation for structural dynamics". ASCE Journal of the Engineering Mechanics Division, 85, pp. 67-94.

[31] Hilber, H., Hughes, T., and Taylor, R., 1977. "Improved numerical dissipation for time integration algorithms in structural dynamics". Earthquake Engineering and Structural Dynamics, 5, pp. 283-292.

[32] Chung, J., and Hulbert, G., 1993. "Time integration algorithm for structural dynamics with improved numerical dissipation: the generalized- $\alpha$ method". ASME Journal of Applied Mechanics, 60(2), pp. $371-375$.

[33] Laursen, T., 2002. Computational Contact and Impact Mechanics. Springer: Berlin, Heidelberg.

[34] Bauchau, O., 1999. "On the modeling of friction and rolling in flexible multi-body systems". Multibody System Dynamics, 3, pp. 209-239. 
[35] Lens, E., Cardona, A., and Géradin, M., 2004. "Energy preserving time integration for constrained multibody systems". Multibody System Dynamics, 11(1), pp. 41-61.

[36] Krause, R., and Walloth, M., 2009. "A time discretization scheme based on Rothe's method for dynamical contact problems with friction". Computer Methods in Applied Mechanics and Engineering, 199, pp. 119.

[37] Krause, R., and Walloth, M., 2012. "Presentation and comparison of selected algorithms for dynamic contact based on the Newmark scheme". Applied Numerical Mathematics, 62, pp. 1393-1410.

[38] Krause, R., and Walloth, M., 2011. "A family of space-time connecting discretization schemes for elastodynamic contact problems with local impact detection". International Journal for Numerical Methods in Engineering, 200, pp. 3425-3438.

[39] Brüls, O., Acary, V., and Cardona, A., 2014. "Simultaneous enforcement of constraints at position and velocity levels in the nonsmooth generalized- $\alpha$ scheme". Computer Methods in Applied Mechanics and Engineering, 281, pp. 131-161.

[40] Acary, V., 2016. "Energy conservation and dissipation properties of time-integration methods for nonsmooth elastodynamics with contact". Journal of Applied Mathematics and Mechanics / Zeitschrift für Angewandte Mathematik und Mechanik, 96(5), May, pp. 585-603.

[41] Cosimo, A., Galvez, J., Cavalieri, F. J., Cardona, A., and Brüls, O., 2019. "A robust nonsmooth generalized- $\alpha$ scheme for flexible systems with impacts". Multibody System Dynamics.

[42] Puso, M. A., and Laursen, T. A., 2004. "A mortar segment-to-segment frictional contact method for large deformations". Computer Methods in Applied Mechanics and Engineering, 193(45), pp. $4891-4913$.

[43] Cavalieri, F. J., and Cardona, A., 2015. "Numerical solution of frictional contact problems based on a mortar algorithm with an augmented lagrangian technique". Multibody System Dynamics, 35(4), Dec, pp. 353375 .

[44] Gear, C., Leimkuhler, B., and Gupta, G., 1985. "Automatic integration of Euler-Lagrange equations with constraints". Journal of Computational and Applied Mathematics, 12-13, pp. 77-90.

[45] Chen, Q.-z., Acary, V., Virlez, G., and Brüls, O., 2013. "A nonsmooth generalized- $\alpha$ scheme for flexible multibody systems with unilateral con- 
straints". International Journal for Numerical Methods in Engineering, 96, pp. 487-511.

[46] Brüls, O., Acary, V., and Cardona, A., 2018. "On the constraints formulation in the nonsmooth generalized- $\alpha$ method". In Advanced Topics in Nonsmooth Dynamics. Springer International Publishing, pp. 335-374.

[47] Martin, A., and Brüls, O., 2007. "Convergence of the generalized- $\alpha$ scheme for constrained mechanical systems". Multibody System Dynamics, 18(2), pp. 185-202.

[48] Cardona, A., and Géradin, M., 1994. "Numerical integration of second order differential - algebraic systems in flexible mechanism dynamics". In Computer-Aided Analysis of Rigid and Flexible Mechanical Systems. Springer Netherlands, pp. 501-529.

[49] Bottasso, C., Bauchau, O., and Cardona, A., 2007. "Time-step-sizeindependent conditioning and sensitivity to perturbations in the numerical solution of index three differential algebraic equations". SIAM J. Scientific Computing, 29, pp. 397-414.

[50] Brüls, O., Cardona, A., and Arnold, M., 2012. "Lie group generalized- $\alpha$ time integration of constrained flexible multibody systems". Mechanism and Machine Theory, 48, pp. $121-137$.

[51] Rockafellar, R., 1976. "Augmented Lagrangians and applications of the proximal point algorithm in convex programming.". Math. Oper. Res., 1, pp. $97-116$.

[52] Zander, R., Forg, M., and Ulbrich, H., 2007. "Impacts on beam structures: Interactions of wave propagation and global dynamics". IUTAM Symposium on Multiscale Problems in Multibody System Contacts, 1, pp. 327-338.

[53] Glocker, C., 2006. "An Introduction to Impacts". In Nonsmooth Mechanics of Solids, J. Haslinger and G. E. Stavroulakis, eds., Vol. 485. Springer Vienna, Vienna, pp. 45-101.

[54] Foerg, M., Geier, T., Neumann, L., and Ulbrich, H., 2006. "r-factor strategies for the augmented Lagrangian approach in multi-body contact mechanics". III European Conference on Computational Mechanics(June), p. 316 .

[55] García Orden, J., and Goicolea, J., 2000. "Conserving properties in constrained dynamics of flexible multibody systems". Multibody System Dynamics, 4(4), pp. 225-244. 
[56] Lens, E., and Cardona, A., 2008. "A nonlinear beam element formulation in the framework of an energy preserving time integration scheme for constrained multibody systems dynamics". Computers and Structures, 86(1), pp. 47-63. 